\title{
Mini-BFSS matrix model in silico
}

\author{
Tarek Anous $\oplus^{1,2, *}$ and Cameron Cogburn ${ }^{3, \dagger}$ \\ ${ }^{1}$ Department of Physics and Astronomy, University of British Columbia, 6224 Agricultural Road, \\ Vancouver, British Columbia V6T 1Z1, Canada \\ ${ }^{2}$ Center for Theoretical Physics, Massachusetts Institute of Technology, \\ Cambridge, Massachusetts 02139, USA \\ ${ }^{3}$ Department of Physics, Massachusetts Institute of Technology, Cambridge, Massachusetts 02139, USA
}

(Received 16 July 2019; published 23 September 2019)

\begin{abstract}
We study a mass-deformed $\mathcal{N}=4$ version of the Banks-Fischler-Shenker-Susskind (BFSS) matrix model with three matrices and gauge group $S U(2)$. This model has zero Witten index. Despite this, we give numerical evidence for the existence of four supersymmetric ground states, two bosonic and two fermionic, in the limit where the mass deformation is tuned to zero.
\end{abstract}

DOI: $10.1103 /$ PhysRevD.100.066023

\section{INTRODUCTION}

This paper concerns itself with the supersymmetric quantum mechanics of three bosonic $S U(N)$ matrices and their fermionic superpartners. The model in question, introduced in [1-3], has four supercharges and describes the low energy effective dynamics of a stack of $N$ wrapped D-branes in a string compactification down to $3+1$ dimensions. When the compactification manifold has curvature and carries magnetic fluxes, the bosonic matrices obtain masses [3]. When the compact manifold is CalabiYau and carries no fluxes, the matrices are massless.

This theory has flat directions whenever the matrices are massless, and hence is a simplified version of the BanksFischler-Shenker-Susskind (BFSS) matrix model [4], which, for the sake of comparison, has nine bosonic $S U(N)$ matrices and 16 supercharges and describes the non-Abelian geometry felt by $D$ particles in a noncompact $(9+1)$-dimensional spacetime. We hence dub the model studied here: mini-BFSS (or mini-BMN [5], for BerensteinMaldacena-Nastase, in the massive case). The Witten index $W_{I}$ has been computed for mini-BFSS [6-10] and vanishes, meaning that the existence of supersymmetric ground states is still an open question. Even the refined index, twisted by a combination of global symmetries and calculated in [9], gives us little information about the set of ground states due to the subtleties associated with computing such indices in

\footnotetext{
tarek@phas.ubc.ca

†cogburn@mit.edu
}

Published by the American Physical Society under the terms of the Creative Commons Attribution 4.0 International license. Further distribution of this work must maintain attribution to the author(s) and the published article's title, journal citation, and DOI. Funded by SCOAP. the presence of flat directions in the potential. This is in stark contrast with the full BFSS model, whose Witten index $W_{I}=1$, implying beyond doubt the existence of at least one supersymmetric ground state. The zero index result for mini-BFSS has led to the interpretation that it may not have any zero energy ground states $[6,10]$, and hence no holographic interpretation. The logic being that, without a rich low energy spectrum, scattering in mini-BFSS would not mimic supergraviton scattering in a putative supersymmetric holographic dual [11]. Of course a vanishing $W_{I}$ does not confirm the absence of supersymmetric ground states-as there may potentially be an exact degeneracy between the bosonic and fermionic states at zero energy.

We weigh in on the existence of supersymmetric states in mini-BFSS by solving the Schrödinger equation numerically for the low-lying spectrum of the $N=2$ model, in the in silico spirit of [12]. To deal with the flat directions we numerically diagonalize the Hamiltonian of the massdeformed mini-BMN matrix model, for which the flat directions are absent, and study the bound state energies as a function of the mass. A numerical analysis of mini-BFSS can also be found in $[13,14]$, which use different methods.

What we uncover is quite surprising. As we tune the mass parameter $m$ to zero, we find evidence for four supersymmetric ground states, two bosonic and two fermionic, which cancel in the evaluation of $W_{I}$. This result seems to agree with plots found in $[13,14]$. It must be said that our result does not constitute an existence proof for supersymmetric threshold bound states in the massless limit, but certainly motivates a further study of the lowlying spectrum of these theories. We reiterate that the Witten index of mini-BFSS vanishes at any $N$, and while we have only given evidence for the existence of zeroenergy states at $N=2$, this encourages us to determine if a similar cancellation between bosonic and fermionic states 
occurs for $N \gg 2$. If this turns out to be the case, it would provide a new addition to the exceedingly small list of matrix models with a potential holographic limit at large $N$.

The organization of the paper is as follows: in Sec. II we present the supercharges, Hamiltonian and symmetry generators of the mini-BMN model for arbitrary $N$. In Sec. III we restrict to $N=2$ and give coordinates in which the Schrödinger equation becomes separable. In Sec. IV we provide our numerical results, and in Sec. V we derive the one-loop effective theory on the moduli space in the massless theory. We conclude with implications for the large- $N$ mini-BFSS model in Sec. VI. We collect formulas for the Schrödinger operators maximally reduced via symmetries in Appendix A and compute the one-loop metric on the Coulomb branch moduli space in Appendix B.

\section{SETUP}

\section{A. Supercharges and Hamiltonian}

Let us consider the supersymmetric quantum mechanics of $S U(N)$ bosonic matrices $X_{A}^{i}$ and their superpartners $\lambda_{A \alpha}$. The quantum mechanics we have in mind has four supercharges ${ }^{1}$ :

$$
\begin{aligned}
& Q_{\alpha}=\left(-i \partial_{X_{A}^{i}}-i m X_{A}^{i}-i W_{A}^{i}\right) \boldsymbol{\sigma}_{\alpha}^{i \gamma} \lambda_{A \gamma}, \\
& \bar{Q}^{\beta}=\bar{\lambda}_{A}^{\gamma} \boldsymbol{\sigma}_{\gamma}^{i \beta}\left(-i \partial_{X_{A}^{i}}+i m X_{A}^{i}+i W_{A}^{i}\right) .
\end{aligned}
$$

The parameter $m$ is simply the mass of $X_{A}^{i}$. The massless version of this model was introduced in [1] and can be derived by dimensionally reducing $\mathcal{N}=1, d=4$ super Yang-Mills to the quantum mechanics of its zero modes. The mass deformation was introduced in [3] and can be obtained from a dimensional reduction of the same gauge theory on $\mathbb{R} \times S^{3}$. We direct the reader to $[2,3]$ for an introduction to these models. This quantum mechanics should be thought of as a simplified version of the BMN matrix model [5] (mini-BMN for brevity). The massless limit should then be thought of as a mini-BFSS matrix model [4]. The lowercase index $i=1, \ldots, 3$ runs over the spatial dimensions (in the language of the original gauge theory), and the uppercase index $A=1, \ldots, N^{2}-1$ runs over the generators of the gauge group $S U(N)$. The $\boldsymbol{\sigma}^{i}$ are the Pauli matrices, and greek indices run over $\alpha=1,2$. In keeping with [1], we have defined $W_{A}^{i} \equiv \partial W / \partial X_{A}^{i}$ where

$$
W \equiv \frac{g}{6} f_{A B C} \epsilon_{i j k} X_{A}^{i} X_{B}^{j} X_{C}^{k}
$$

\footnotetext{
${ }^{1}$ Spinors and their conjugates transform, respectively, in the $\mathbf{2}$ and $\overline{2}$ of $S O(3)$. Spinor indices are raised and lowered using the Levi-Civita symbol $\epsilon^{\alpha \beta}=-\epsilon_{\alpha \beta}$ with $\epsilon^{12}=1$. Thus in our
} conventions

$$
(\bar{\psi} \epsilon)_{\alpha}=\bar{\psi}^{\gamma} \epsilon_{\gamma \alpha}, \quad(\epsilon \psi)^{\alpha}=\epsilon^{\alpha \gamma} \psi_{\gamma}, \quad \epsilon_{\alpha \omega} \epsilon^{\omega \beta}=\delta_{\alpha}^{\beta} .
$$

and $f_{A B C}$ are the structure constants of $S U(N)$. The gauginos obey the canonical fermionic commutation relations $\left\{\lambda_{A \alpha}, \bar{\lambda}_{B}^{\beta}\right\}=\delta_{A B} \delta_{\alpha}^{\beta}$, and hence the algebra generated by these supercharges is [3]

$$
\begin{aligned}
& \left\{Q_{\alpha}, \bar{Q}^{\beta}\right\}=2\left(\delta_{\alpha}^{\beta} H-g \sigma_{\alpha}^{k \beta} X_{A}^{k} G_{A}+m \sigma_{\alpha}^{k \beta} J^{k}\right), \\
& \left\{\bar{Q}^{\alpha}, \bar{Q}^{\beta}\right\}=\left\{Q_{\alpha}, Q_{\beta}\right\}=0
\end{aligned}
$$

with Hamiltonian

$$
\begin{aligned}
H \equiv & -\frac{1}{2} \partial_{X_{A}^{i}} \partial_{X_{A}^{i}}+\frac{1}{2} m^{2}\left(X_{A}^{i}\right)^{2}+m X_{A}^{i} W_{A}^{i} \\
& +\frac{g^{2}}{4}\left(f_{A B C} X_{B}^{i} X_{C}^{j}\right)^{2}-\frac{3}{4} m\left[\bar{\lambda}_{A}, \lambda_{A}\right]+i g f_{A B C} \bar{\lambda}_{A} X_{B}^{k} \boldsymbol{\sigma}^{k} \lambda_{C} .
\end{aligned}
$$

The operators $G_{A}$ and $J^{k}$ appearing in the algebra are, respectively, the generators of gauge transformations and $S O(3)$ rotations. These are given by

$$
\begin{aligned}
G_{A} & \equiv-i f_{A B C}\left(X_{B}^{i} \partial_{X_{C}^{i}}+\bar{\lambda}_{B} \lambda_{C}\right), \\
J^{i} & \equiv-i \epsilon_{i j k} X_{A}^{j} \partial_{X_{A}^{k}}+\frac{1}{2} \bar{\lambda}_{A} \sigma^{i} \lambda_{A} .
\end{aligned}
$$

In solving for the spectrum of this theory, we must impose the constraint $G_{A}|\psi\rangle=0, \forall A$. In the above expressions, whenever fermionic indices are suppressed, it implies that they are being summed over.

Let us briefly note the dimensions of the fields and parameters in units of the energy $[\mathcal{E}]=1$. These are $[X]=-1 / 2,[\lambda]=0,[g]=3 / 2$, and $[m]=1$. Therefore, an important role will be played by the dimensionless quantity

$$
\nu \equiv \frac{m}{g^{2 / 3}} .
$$

We consider here the mass deformed gauge quantum mechanics because, in the absence of the mass parameter $m$, the classical potential has flat directions (see Fig. 1). Turning on this mass deformation gives us a dimensionless parameter $\nu$, to tune in studying the spectrum of this theory, and allows us to approach the massless limit from above.

\section{B. Symmetry algebra}

Let us now give the symmetry algebra of the theory. The components of $\vec{J}$ satisfy

$$
\begin{aligned}
& {\left[J^{i}, J^{j}\right]=i \epsilon_{i j k} J^{k}, \quad\left[J^{i}, Q_{\alpha}\right]=-\frac{1}{2} \boldsymbol{\sigma}_{\alpha}^{i \gamma} Q_{\gamma},} \\
& {\left[\vec{J}^{2}, J^{i}\right]=0, \quad\left[J^{i}, \bar{Q}^{\alpha}\right]=\frac{1}{2} \bar{Q}^{\beta} \boldsymbol{\sigma}_{\beta}^{i \alpha} .}
\end{aligned}
$$

There is an additional $U(1)_{R}$ generator $R \equiv \bar{\lambda}_{A} \lambda_{A}$ which counts the number of fermions. It satisfies 


$$
\left[R, Q_{\alpha}\right]=-Q_{\alpha}, \quad\left[R, \bar{Q}^{\alpha}\right]=+\bar{Q}^{\alpha}, \quad\left[R, J^{i}\right]=0 .
$$

The Hamiltonian also has a particle-hole symmetry,

$\bar{\lambda}_{A}^{\alpha} \rightarrow \epsilon^{\alpha \gamma} \lambda_{A \gamma}, \quad \lambda_{A \alpha} \rightarrow \bar{\lambda}_{A}^{\gamma} \epsilon_{\gamma \alpha}, \quad \epsilon^{12}=-\epsilon_{12}=1$,

where $\epsilon^{\alpha \beta}$ is the Levi-Civita symbol. This transformation leaves the Hamiltonian invariant but takes $R \rightarrow$ $2\left(N^{2}-1\right)-R$ and effectively cuts our problem in half.

One peculiar feature of the mass deformed theory is that the supercharges do not commute with the Hamiltonian as a result of the vector $\vec{J}$ appearing in (2.4). It is easy to show that

$$
\left[H, Q_{\alpha}\right]=\frac{m}{2} Q_{\alpha}, \quad\left[H, \bar{Q}^{\beta}\right]=-\frac{m}{2} \bar{Q}^{\beta} .
$$

Thus, acting with a supercharge increases/decreases the energy of a state by $\pm \frac{m}{2}$. This is a question of $R$ frames, as discussed in [3]. Essentially we can choose to measure energies with respect to the shifted Hamiltonian $H_{m} \equiv$ $H+\frac{m}{2} R$, which commutes with the supercharges, and write the algebra as

$\left\{Q_{\alpha}, \bar{Q}^{\beta}\right\}=2\left\{\delta_{\alpha}^{\beta}\left(H_{m}-\frac{m}{2} R\right)-g \boldsymbol{\sigma}_{\alpha}^{k \beta} X_{A}^{k} G_{A}+m \boldsymbol{\sigma}_{\alpha}^{k \beta} \boldsymbol{J}^{k}\right\}$.

\section{Interpretation as $D$ particles}

The $\nu \rightarrow 0$ limit of this model can be thought of as the world volume theory of a stack of $N$ D-branes compactified along a special Lagrangian cycle of a Calabi-Yau threefold [2]. The $X_{A}^{i}$ then parametrize the non-Abelian geometry felt by the compactified $D$ particles in the remaining noncompact $(3+1)$-dimensional asymptotically flat spacetime. The addition of the mass parameter corresponds to adding curvature and magnetic fluxes to the compact manifold [3], changing the asymptotics of the noncompact spacetime to $\mathrm{AdS}_{4}$. This interpretation was argued in [3,15] and passes several consistency checks. Hence we should think of the mass deformed theory as describing the nonrelativistic dynamics of $D$ particles in an asymptotically $\mathrm{AdS}_{4}$ spacetime and the massless limit as taking the AdS radius to infinity in units of the string length.

To be more specific, it will be useful to translate between our conventions and the conventions of [3]. One identifies $m=\Omega, g^{2}=1 / m_{v},\{X, \lambda\}_{\text {us }}=m_{v}^{1 / 2}\{X, \lambda\}_{\text {them }}$ in units where the string length $l_{s}=1$. Reintroducing $l_{s}$, this dictionary implies that $g^{2}=g_{s} / l_{s}^{3} \sqrt{2 \pi}$, with $g_{s}$ the string coupling, gets set by a combination of the magnetic fluxes threading the compact manifold, and similarly $\ell_{\text {AdS }} \equiv 1 / m$ gets set by a combination of these magnetic fluxes and the string length. For $\mathrm{AdS}_{4} \times \mathbb{C} P^{3}$ compactifications dual to the Aharony-Bergman-Jafferis-Maldacena (ABJM) theory this was worked out in detail in $[3,16]$, and they identify

$$
g_{s}=\left(\frac{32 \pi^{2} N}{k^{5}}\right)^{\frac{1}{4}}, \quad \ell_{\mathrm{AdS}}=\left(\frac{N}{8 \pi^{2} k}\right)^{\frac{1}{4}} l_{s}
$$

where $k$ and $N$ are, respectively, integrally quantized magnetic 2-form and 6-form flux. In this example taking $\nu=\sqrt{2 \pi}\left(k^{2} / N\right)^{1 / 3} \rightarrow 0$ while keeping $g_{s}$ fixed takes the AdS radius to infinity in units of $l_{s}$.

The main focus of the next sections is on whether this stack of $D$ particles forms a supersymmetric bound state, particularly in the $\nu \rightarrow 0$ limit. There the Witten index $W_{I} \equiv \operatorname{Tr}_{\mathcal{H}}\left\{(-1)^{R} e^{-\beta H}\right\}$ has been computed [6-10] and evaluates to zero. This is in contrast with the full BFSS matrix model, whose index is $W_{I}=1$, confirming the existence of a supersymmetric ground state. We will use the numerical approach of [12] and verify if supersymmetry is preserved or broken in the $S U(2)$ case. We find evidence that supersymmetry is preserved in the $\nu \rightarrow 0$ limit, and that there are precisely four ground states contributing to the vanishing Witten index.

\section{QUANTIZING THE $S U(2)$ THEORY}

\section{A. Polar representation of the matrices}

We are aiming to solve the Schrödinger problem $H_{m}|\psi\rangle=\mathcal{E}_{m}|\psi\rangle$. We will not be able to do this for arbitrary $N$, and from here on we will restrict to gauge group $S U(2)$ for which the structure constants $f_{A B C}=\epsilon_{A B C}$. In this case the wave functions depend on 9 bosonic degrees of freedom tensored into a 64-dimensional fermionic Hilbert space. It is thus incumbent upon us to reduce this problem maximally via symmetry. To do so, we exploit the fact that the matrices $X_{A}^{i}$ admit a polar decomposition as follows:

$$
X_{A}^{i}=L_{A B} \Lambda_{B}^{j} M^{\mathrm{T} j i},
$$

where

$L \equiv e^{-i \varphi_{1} \mathcal{L}^{3}} e^{-i \varphi_{2} \mathcal{L}^{2}} e^{-i \varphi_{3} \mathcal{L}^{3}}, \quad M \equiv e^{-i \vartheta_{1} \mathcal{L}^{3}} e^{-i \vartheta_{2} \mathcal{L}^{2}} e^{-i \vartheta_{3} \mathcal{L}^{3}}$,

and $\left[\mathcal{L}^{i}\right]_{j k} \equiv-i \epsilon_{i j k}$ are the generators of $S O(3)$. The diagonal matrix

$$
\Lambda \equiv \operatorname{diag}\left(\mathbf{x}_{1}, \mathbf{x}_{2}, \mathbf{x}_{3}\right)
$$

represents the spatial separation between the pair of D-branes in the stack. The $\varphi_{i}$ and $\vartheta_{i}$ represent the (respectively, gauge-dependent and gauge-independent) Euler-angle rigid body rotations of the configuration space. 
This parametrization is useful because the Schrödinger equation is separable in these variables, as we show in Appendix A.

The metric on configuration space can be reexpressed as

$\sum_{A, i} d X_{A}^{i} d X_{A}^{i}=\sum_{a=1}^{3} d \mathbf{x}_{a}^{2}+I_{a}\left(d \Omega_{a}^{2}+d \omega_{a}^{2}\right)-2 K_{a} d \Omega_{a} d \omega_{a}$,

$$
I_{a} \equiv \mathbf{x}_{b} \mathbf{x}_{b}-\mathbf{x}_{a}^{2}, \quad K_{a} \equiv\left|\epsilon_{a b c}\right| \mathbf{x}_{b} \mathbf{x}_{c} .
$$

The angular differentials are the usual $S U(2)$ CartanMaurer differential forms defined as follows:

$d \omega_{a}=-\frac{1}{2} \epsilon_{a b c}\left[L^{T} \cdot d L\right]_{b c}, \quad d \Omega_{a}=-\frac{1}{2} \epsilon_{a b c}\left[M^{T} \cdot d M\right]_{b c}$.

The volume element used to compute the norm of the wave function is

$$
\prod_{i, A} d X_{A}^{i} d X_{A}^{i}=\Delta\left(\mathbf{x}_{a}\right) \prod_{i=1}^{3} d \mathbf{x}_{i} \sin \varphi_{2} \prod_{j=1}^{3} d \varphi_{j} \sin \vartheta_{2} \prod_{k=1}^{3} d \vartheta_{k},
$$

where $\Delta\left(\mathbf{x}_{a}\right) \equiv\left(\mathbf{x}_{1}^{2}-\mathbf{x}_{2}^{2}\right)\left(\mathbf{x}_{3}^{2}-\mathbf{x}_{2}^{2}\right)\left(\mathbf{x}_{3}^{2}-\mathbf{x}_{1}^{2}\right)$ is the Vandermonde determinant with squared eigenvalues. To cover the configuration space correctly, we take the new coordinates to lie in the range [17]

$$
\begin{aligned}
& \mathbf{x}_{3} \geq \mathbf{x}_{1} \geq\left|\mathbf{x}_{2}\right| \geq 0, \quad \pi \geq \varphi_{2}, \vartheta_{2} \geq 0, \\
& 2 \pi \geq \varphi_{i \neq 2}, \vartheta_{i \neq 2} \geq 0 .
\end{aligned}
$$

The generators of gauge transformations $G_{A}$ and rotations $J^{i}$ are given in (2.6). These satisfy

$$
\begin{aligned}
& {\left[J^{i}, J^{j}\right]=i \epsilon_{i j k} J^{k}, \quad\left[G_{A}, G_{B}\right]=i \epsilon_{A B C} G_{C},} \\
& {\left[\vec{J}^{2}, J^{i}\right]=0, \quad\left[J^{i}, G_{A}\right]=0 .}
\end{aligned}
$$

To label the $S U(2)_{\text {gauge }} \times S O(3)_{J}$ representations of the wave functions, it is useful to define the "body fixed" angular momentum and gauge operators $\vec{P} \equiv M^{-1} \cdot \vec{J}$ and $\vec{S} \equiv L^{-1} \cdot \vec{G}$, which satisfy

$$
\begin{aligned}
\vec{P}^{2} & =\vec{J}^{2}, \quad \vec{S}^{2}=\vec{G}^{2}, \\
{\left[P^{i}, P^{j}\right] } & =-i \epsilon_{i j k} P^{k}, \quad\left[S_{A}, S_{B}\right]=-i \epsilon_{A B C} S_{C}, \\
{\left[P^{i}, J^{j}\right] } & =0, \quad\left[S_{A}, G_{B}\right]=0 .
\end{aligned}
$$

Unlike the generators of angular momentum, $\vec{P}$ is not conserved. However, as we explain in Appendix A, it is still useful for separating variables.
Let us give expressions for the bosonic parts of $\vec{J}$ and $\vec{P}$, which we call $\overrightarrow{\mathcal{J}}$ and $\overrightarrow{\mathcal{P}}$, respectively, in terms of the angular coordinates. These are

$$
\mathcal{J}^{1}=-i\left(-\cos \vartheta_{1} \cot \vartheta_{2} \partial_{\vartheta_{1}}-\sin \vartheta_{1} \partial_{\vartheta_{2}}+\frac{\cos \vartheta_{1}}{\sin \vartheta_{2}} \partial_{\vartheta_{3}}\right),
$$

$\mathcal{J}^{2}=-i\left(-\sin \vartheta_{1} \cot \vartheta_{2} \partial_{\vartheta_{1}}+\cos \vartheta_{1} \partial_{\vartheta_{2}}+\frac{\sin \vartheta_{1}}{\sin \vartheta_{2}} \partial_{\vartheta_{3}}\right)$,

$$
\mathcal{J}^{3}=-i \partial_{\vartheta_{1}}
$$

and

$\mathcal{P}^{1}=-i\left(-\frac{\cos \vartheta_{3}}{\sin \vartheta_{2}} \partial_{\vartheta_{1}}+\sin \vartheta_{3} \partial_{\vartheta_{2}}+\cot \vartheta_{2} \cos \vartheta_{3} \partial_{\vartheta_{3}}\right)$,

$$
\mathcal{P}^{2}=-i\left(\frac{\sin \vartheta_{3}}{\sin \vartheta_{2}} \partial_{\vartheta_{1}}+\cos \vartheta_{3} \partial_{\vartheta_{2}}-\cot \vartheta_{2} \sin \vartheta_{3} \partial_{\vartheta_{3}}\right),
$$

$$
\mathcal{P}^{3}=-i \partial_{\vartheta_{3}} .
$$

Similarly let us define $\mathcal{G}_{A}$ and $\mathcal{S}_{A}$ as the bosonic parts of the $G_{A}$ and $S_{A}$ operators. The $\mathcal{G}_{A}$ are related to the $\mathcal{J}^{i}$ by replacing $\vartheta_{i} \rightarrow \varphi_{i}$. It is easy to guess that the $\mathcal{S}_{A}$ are then related to the $\mathcal{P}^{i}$ via the same replacement.

We are now ready to give expressions for the momentum operators and the kinetic energy operator in terms of the new variables. These are [18]

$$
-i \partial_{X_{A}^{i}}=-i L_{A a} M^{i b}\left\{\delta_{a b} \partial_{\mathbf{x}_{a}}+i \frac{\epsilon_{a b c}}{\mathbf{x}_{a}^{2}-\mathbf{x}_{b}^{2}}\left(\mathbf{x}_{a} \mathcal{P}^{c}+\mathbf{x}_{b} \mathcal{S}_{c}\right)\right\},
$$

$$
\begin{aligned}
-\frac{1}{2} \partial_{X_{A}^{i}} \partial_{X_{A}^{i}}= & -\frac{1}{2 \Delta} \partial_{\mathbf{x}_{a}} \Delta \partial_{\mathbf{x}_{a}} \\
& +\frac{1}{2} \sum_{a=1}^{3} \frac{I_{a}\left(\mathcal{P}^{a 2}+\mathcal{S}_{a}^{2}\right)+2 K_{a} \mathcal{P}^{a} \mathcal{S}_{a}}{I_{a}^{2}-K_{a}^{2}} .
\end{aligned}
$$

It is also straightforward to write down the bosonic potential $V$ in terms of the new variables:

$$
V=\frac{1}{2} m^{2} \mathbf{x}_{a} \mathbf{x}_{a}+3 g m \mathbf{x}_{1} \mathbf{x}_{2} \mathbf{x}_{3}+\frac{g^{2}}{2}\left(\mathbf{x}_{1}^{2} \mathbf{x}_{2}^{2}+\mathbf{x}_{1}^{2} \mathbf{x}_{3}^{2}+\mathbf{x}_{2}^{2} \mathbf{x}_{3}^{2}\right) .
$$



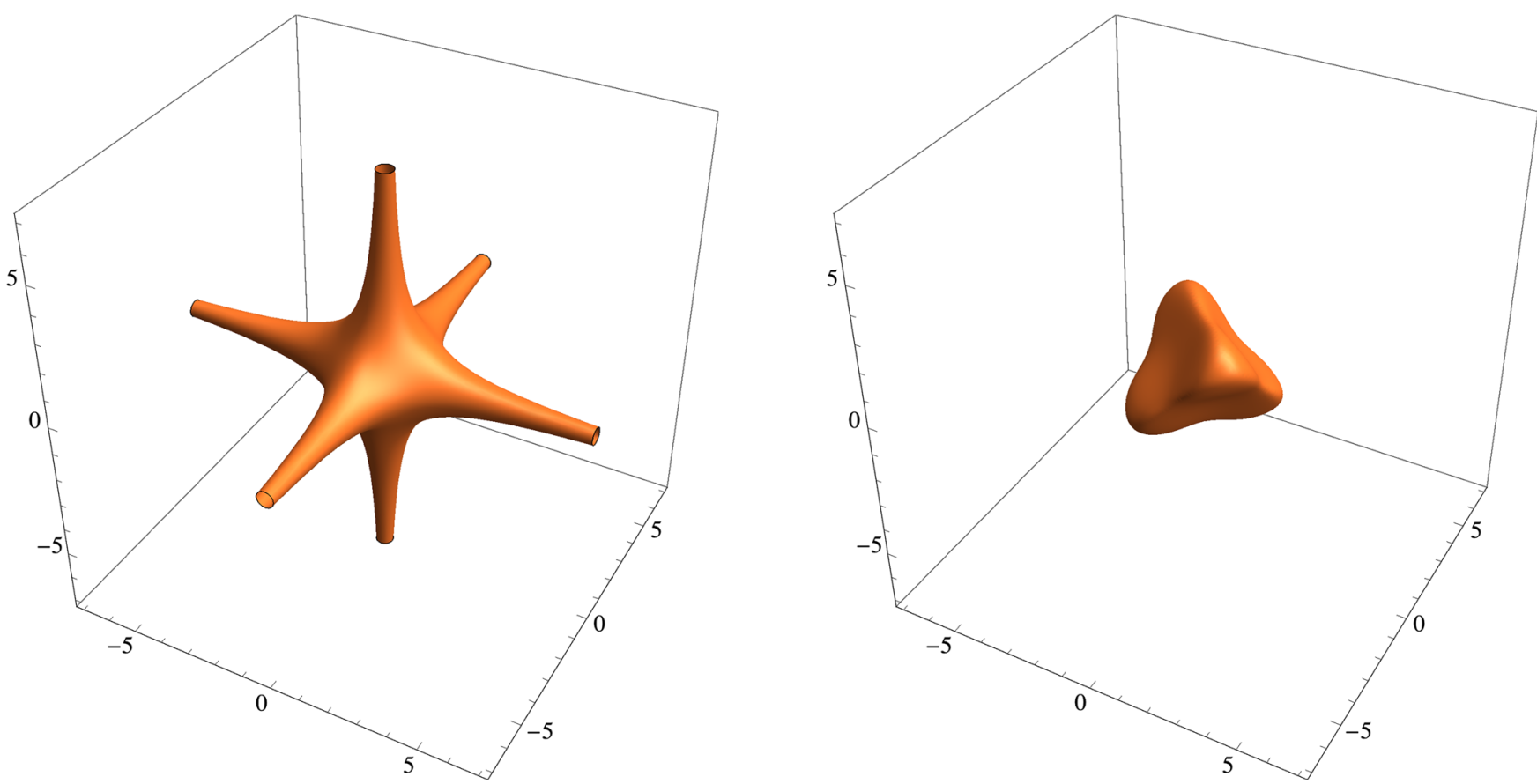

FIG. 1. Contours of constant potential energy $V=2$ in units where $g=1$ as a function of $\mathbf{x}_{a}$. The left-hand figure is evaluated at $m=0$, whereas the right-hand figure is evaluated at $m=1$. The long spikes in the left figure are indicative of the flat directions along the moduli space. These flat directions get lifted for any finite $m$.

As expected it is independent of the angular variables. We have depicted constant potential surfaces in Fig. 1.

Apart from the coordinates $\mathbf{x}_{a}$ the following nonlinear coordinates will often appear in the equations below:

$$
\begin{aligned}
& \mathbf{y}_{a} \equiv \frac{I_{a}}{I_{a}^{2}-K_{a}^{2}}=\frac{1}{2}\left|\epsilon_{a b c}\right| \frac{\mathbf{x}_{b}^{2}+\mathbf{x}_{c}^{2}}{\left(\mathbf{x}_{b}^{2}-\mathbf{x}_{c}^{2}\right)^{2}}, \\
& \mathbf{z}_{a} \equiv \frac{K_{a}}{I_{a}^{2}-K_{a}^{2}}=\left|\epsilon_{a b c}\right| \frac{\mathbf{x}_{b} \mathbf{x}_{c}}{\left(\mathbf{x}_{b}^{2}-\mathbf{x}_{c}^{2}\right)^{2}} .
\end{aligned}
$$

With these definitions the kinetic term can be written as

$$
\begin{aligned}
-\frac{1}{2} \partial_{X_{A}^{i}} \partial_{X_{A}^{i}}= & -\frac{1}{2 \Delta} \partial_{\mathbf{x}_{a}} \Delta \partial_{\mathbf{x}_{a}} \\
& +\frac{1}{2}\left[\mathbf{y}_{a}\left(\mathcal{P}^{a 2}+\mathcal{S}_{a}^{2}\right)+2 \mathbf{z}_{a} \mathcal{P}^{a} \mathcal{S}_{a}\right]
\end{aligned}
$$

Notice that the term $\sum_{a=1}^{3} \mathbf{y}_{a} \mathcal{P}^{a 2}$ is the kinetic energy of a rigid rotor with principal moments of inertial $\mathbf{y}_{a}^{-1}$. Unlike the $c=1$ matrix model, the angular-independent piece of the kinetic term cannot be trivialized by absorbing a factor of $\sqrt{\Delta}$ into the wave function [19]. Instead, we have

$$
-\frac{1}{2 \Delta} \partial_{\mathbf{x}_{a}} \Delta \partial_{\mathbf{x}_{a}}=-\frac{1}{2}\left(\frac{1}{\sqrt{\Delta}} \partial_{\mathbf{x}_{a}}^{2} \sqrt{\Delta}+T\right),
$$

where

$$
T \equiv \sum_{a=1}^{3} \mathbf{y}_{a}=\frac{\mathbf{x}_{1}^{2}+\mathbf{x}_{2}^{2}}{\left(\mathbf{x}_{1}^{2}-\mathbf{x}_{2}^{2}\right)^{2}}+\frac{\mathbf{x}_{1}^{2}+\mathbf{x}_{3}^{2}}{\left(\mathbf{x}_{1}^{2}-\mathbf{x}_{3}^{2}\right)^{2}}+\frac{\mathbf{x}_{2}^{2}+\mathbf{x}_{3}^{2}}{\left(\mathbf{x}_{3}^{2}-\mathbf{x}_{3}^{2}\right)^{2}},
$$

and its appearance in the Schrödinger equation acts as an attractive effective potential between the $\mathbf{x}_{a}$.

\section{B. Gauge-invariant fermions}

Because the operators $G_{A}$ in (2.6) have a nontrivial dependence on the gauginos $\lambda_{A \alpha}$, it is not sufficient to suppress the wave function's dependence on gauge angles $\varphi_{i}$ entirely. Instead, we can write down a set of gaugeinvariant fermions that will contain the entire dependence on the gauge angles [20]:

$$
\chi_{A \alpha} \equiv L_{B A} \lambda_{B \alpha}, \quad \bar{\chi}_{A}^{\beta} \equiv L_{B A} \bar{\lambda}_{B}^{\beta} .
$$

These satisfy $\left\{\chi_{A \alpha}, \bar{\chi}_{B}^{\beta}\right\}=\delta_{A B} \delta_{\alpha}^{\beta}$, but no longer commute with bosonic derivatives. Defining $\tilde{\boldsymbol{\sigma}}_{\alpha}^{i \beta} \equiv M^{j i} \boldsymbol{\sigma}_{\alpha}^{j \beta}$, we can now write the supercharges in terms of the new parametrization. These are 


$$
\begin{aligned}
& Q_{\alpha}=-i \tilde{\boldsymbol{\sigma}}_{\alpha}^{b \gamma} \chi_{a \gamma}\left(\delta_{a b}\left\{\partial_{\mathbf{x}_{b}}+m \mathbf{x}_{b}+\frac{g}{2}\left|\epsilon_{b s t}\right| \mathbf{x}_{s} \mathbf{x}_{t}\right\}+i \frac{\epsilon_{a b c}}{\mathbf{x}_{a}^{2}-\mathbf{x}_{b}^{2}}\left(\mathbf{x}_{a} \mathcal{P}^{c}+\mathbf{x}_{b} \mathcal{S}_{c}\right)\right), \\
& \bar{Q}^{\beta}=-i \bar{\chi}_{a}^{\gamma} \tilde{\boldsymbol{\sigma}}_{\gamma}^{b \beta}\left(\delta_{a b}\left\{\partial_{\mathbf{x}_{b}}-m \mathbf{x}_{b}-\frac{g}{2}\left|\epsilon_{b s t}\right| \mathbf{x}_{s} \mathbf{x}_{t}\right\}+i \frac{\epsilon_{a b c}}{\mathbf{x}_{a}^{2}-\mathbf{x}_{b}^{2}}\left(\mathbf{x}_{a} \mathcal{P}^{c}+\mathbf{x}_{b} \mathcal{S}_{c}\right)\right),
\end{aligned}
$$

where we have put the gauge-invariant fermions to the left so as to remind the reader that the bosonic derivatives are not meant to act on them in the supercharges. The Hamiltonian $H$ (not $H_{m}$ ) in the new parametrization is

$$
\begin{aligned}
H= & -\frac{1}{2 \Delta} \partial_{\mathbf{x}_{a}} \Delta \partial_{\mathbf{x}_{a}}+\frac{1}{2}\left[\mathbf{y}_{a}\left(\mathcal{P}^{a 2}+\mathcal{S}_{a}^{2}\right)+2 \mathbf{z}_{a} \mathcal{P}^{a} \mathcal{S}_{a}\right] \\
& +\frac{1}{2} m^{2} \mathbf{x}_{a} \mathbf{x}_{a}+3 g m \mathbf{x}_{1} \mathbf{x}_{2} \mathbf{x}_{3} \\
& +\frac{g^{2}}{2}\left(\mathbf{x}_{1}^{2} \mathbf{x}_{2}^{2}+\mathbf{x}_{1}^{2} \mathbf{x}_{3}^{2}+\mathbf{x}_{2}^{2} \mathbf{x}_{3}^{2}\right)-\frac{3}{4} m\left[\bar{\chi}_{A}, \chi_{A}\right] \\
& +i g \epsilon_{A k C} \bar{\chi}_{A} \mathbf{x}_{k} \tilde{\boldsymbol{\sigma}}^{k} \chi_{C} .
\end{aligned}
$$

\section{NUMERICAL RESULTS}

To calculate the spectrum of the Hamiltonian (3.25), we must reduce our problem using symmetry; that is, we should label our states via the maximal commuting set of conserved quantities: $H_{m}, J^{3}, \vec{J}^{2}, R$. Because of the discrete particle-hole symmetry (2.10) we need only consider $R=0, \ldots, 3$. In Appendix $\mathrm{A}$ we construct gaugeinvariant highest-weight representations of $S O(3)_{J}$ in each $R$-charge sector. This means we fix the wave functions' dependence on the angles $\vartheta_{i}$ and $\varphi_{i}$ and provide the reduced Schrödinger operators that depend only on $\mathbf{x}_{a} \cdot{ }^{2}$

Our numerical results for the lowest energy states of $H_{m}$ for each $R$ and $j$ are presented in Fig. 2 and were obtained by inputting the restricted Schrödinger equations of Appendix A into Mathematica's NDEIGENVALUES command, which uses a finite element approach to solve for the eigenfunctions of a coupled differential operator on a restricted domain. We have labeled each row by the fermion number $R$ and each column by the $S O(3)_{J}$ highest weight eigenvalue $j$ (i.e., $\vec{J}^{2}|\psi\rangle=j(j+1)|\psi\rangle$ and $J^{3}|\psi\rangle=j|\psi\rangle$ ).

A few comments are in order:

(1) The most striking feature of these plots is the seeming appearance of zero energy states for $(R, j)=(2,0)$ and $(R, j)=(3,1 / 2)$ as $\quad \nu \rightarrow 0$. Since the Witten index $W_{I}=0$, and since the states in the $(2,0)$ and $(3,1 / 2)$ sectors seem to have nonzero energy for any finite $\nu$, it must be the case that these states are elements of the same supersymmetry multiplet. This must be so for the deformation invariance of $W_{I}$.

\footnotetext{
${ }^{2}$ We only provide a small set of these reduced Schrödinger operators, as they increase in size with increasing $S O(3)_{J}$ eigenvalue $j$.
}

(2) Since we know, by construction, that the lowest energy $(R, j)=(2,0)$ and $(R, j)=(3,1 / 2)$ states are related by supersymmetry, we can use the difference in their numerically obtained energies as a benchmark of our numerical errors. Obtaining the $(R, j)=(2,0)$ ground state energy required solving a coupled Schrödinger equation involving 15 functions in 3 variables. For the $(R, j)=(3,1 / 2)$ state, the number of functions one is numerically solving for jumps to 40 . In the latter case, it was difficult to reduce our error (either by refining the finite element mesh or by increasing the size of the domain) in a significant way without Mathematica crashing. This is despite the fact that we had 12 cores and $64 \mathrm{~Gb}$ of RAM at our disposal. In Fig. 3 we plot the percentage error in the $H_{m}$ energy difference between these two states as a function of $\nu$. We find that the energy difference between these states is around $13 \%$ of the total energy as a function of $\nu$. For comparison, we also do this for the lowest $(R, j)=(0,0)$ and $(R, j)=(1,1 / 2)$ states, where the numerics are more reliable as a result of solving a much simpler set of equations. There the difference between the computed energies is at most $2 \%$.

(3) Our results suggest that there are four supersymmetric states, two of which are bosonic and two of which are fermionic, which would cancel in the evaluation of the index. Explicitly, the two bosonic states are $j=0$ singlets in the $R=2$ and $R=4$ sectors (recall the discrete particle hole symmetry of the theory) and the two fermionic states are the $j=$ $1 / 2$ doublet in the $R=3$ sector. It is interesting to note that there are not more states in this multiplet; for example, numerically studying the $(R, j)=$ $(0,1)$ sector reveals no evidence for a supersymmetric state in the $\nu \rightarrow 0$ limit.

(4) The massless $S U(2)$ model was studied using a different numerical approach in $[13,14]$, and their plots for the ground state energies seem to approach ours, particularly Figs. 2 and 5 of [14].

(5) Our numerical evidence for these supersymmetric states does not constitute a proof since we will never be able to numerically resolve if this state has exactly zero energy. However, the result is highly suggestive of a supersymmetry preserving set of states at $\nu=0$, and there is no contradiction with the analytically obtained Witten index result $W_{I}=0$. It would be interesting to analyze the existence of these states analytically in future work. 

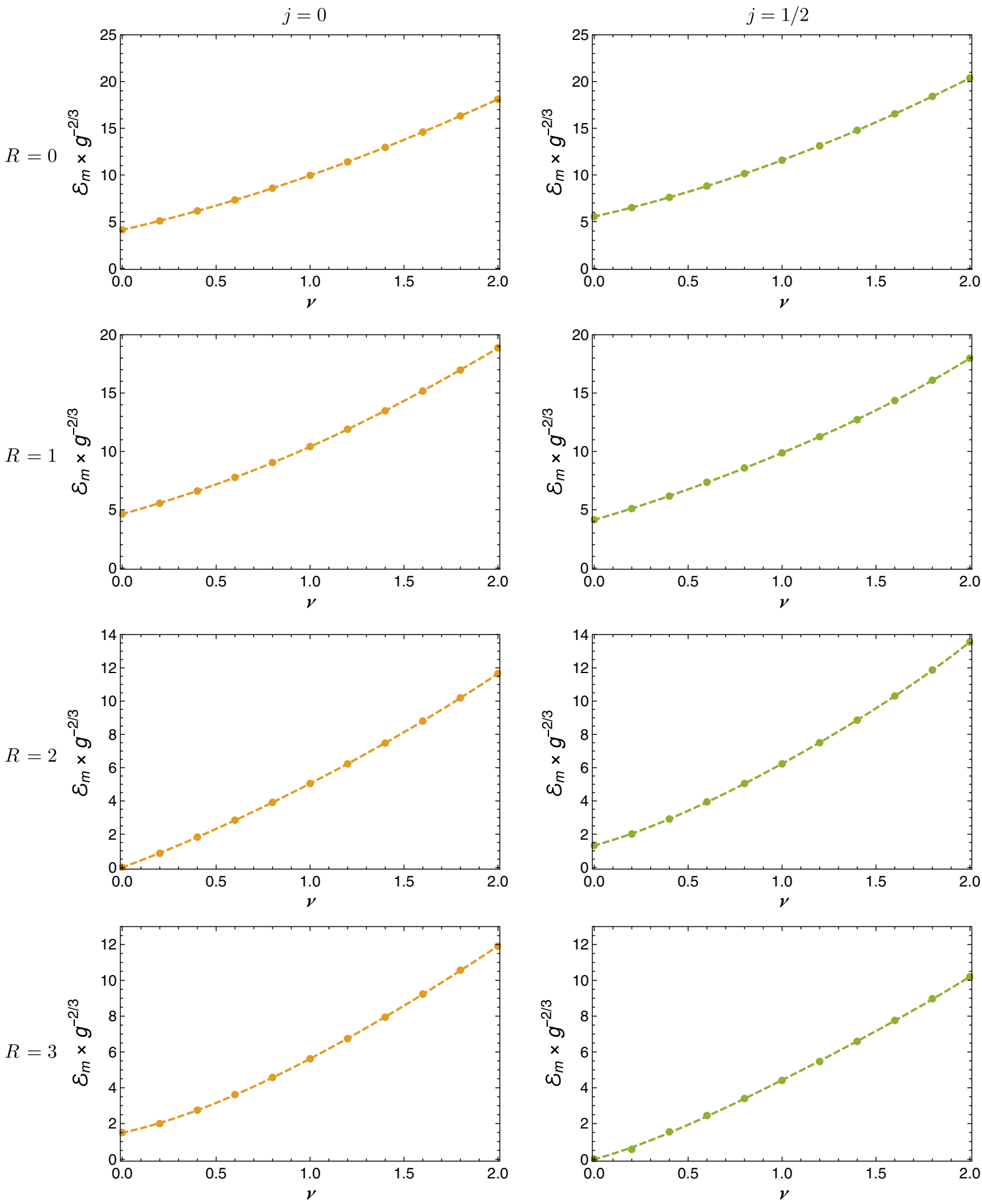

FIG. 2. Lowest energy eigenvalue for $R=\{0,1,2,3\}$ and $j=\{0,1 / 2\}$ as a function of $\nu$. Each row corresponds to a different value of $R$ up to 3 , and the columns are labeled by $j=0$ or $j=1 / 2$. Note that for $\nu=0$ there are $\mathcal{E}=0$ energy eigenstates in both the $R=2$ and $R=3$ sectors of the theory. This implies the existence of four supersymmetric ground states at $\nu=0$, a fermionic $j=1 / 2$ doublet in the $R=3$ sector, and two bosonic $j=0$ singlets in the $R=2$ and $R=4$ sectors. 

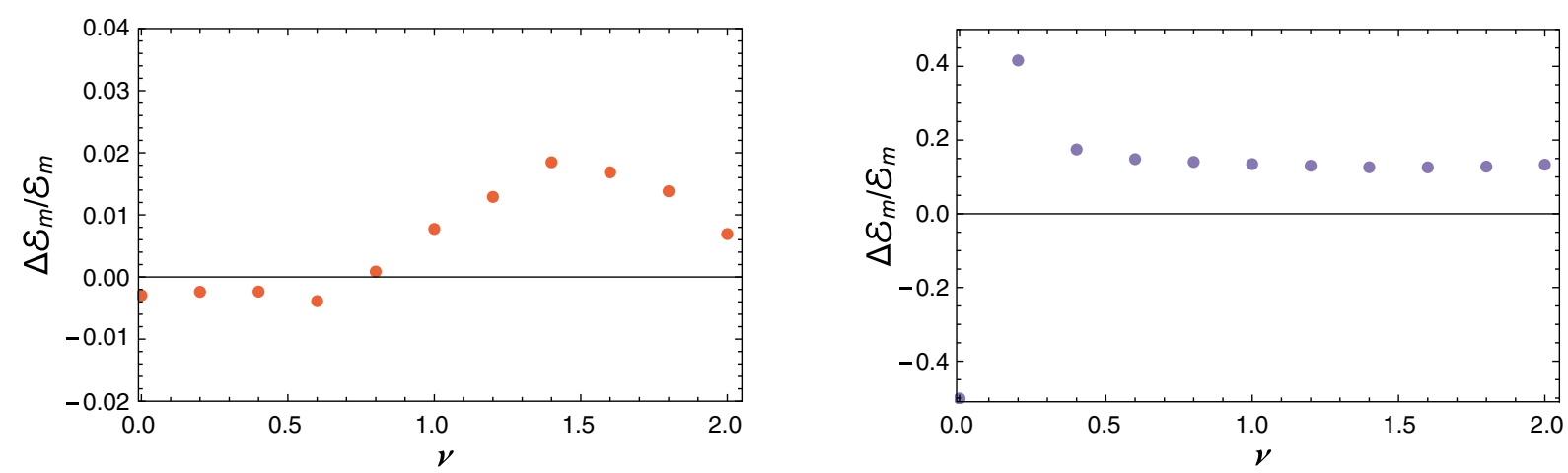

FIG. 3. Left: Percentage energy difference between the lowest $(R, j)=(0,0)$ state and the lowest $(R, j)=(1,1 / 2)$ state. As expected by supersymmetry their energies match to within $2 \%$. Right: Percentage energy difference between the lowest $(R, j)=(2,0)$ state and the lowest $(R, j)=(3,1 / 2)$ state. These energies match to within $13 \%$. The percentage error is larger for low $\nu$ since the energy is approaching zero.

\section{EFFECTIVE THEORY ON THE MODULI SPACE OF THE $S U(2)$ MODEL}

To get a better handle on the previous section's numerical results, we will now study the $\nu \rightarrow 0$ limit of the matrix model analytically. Since the full problem is clearly quite difficult even for $N=2$, we will study the massless model in some parametric limit. This is possible because the theory has a moduli space ${ }^{3}$ - a flat direction where the D-branes can become well separated, and along this moduli space certain fields become massive and can be integrated out. We will parametrize this moduli space by the coordinates $\left(\mathbf{x}_{3}, \vartheta_{2}, \vartheta_{1}\right)$ and will henceforth label them $\left(\mathbf{x}_{3}, \vartheta_{2}, \vartheta_{1}\right) \rightarrow(r, \theta, \phi)$ for the remainder of this section. The parametric limit we will take is the limit of large $r$.

To derive the effective theory along the moduli space, we will first take $(r, \theta, \phi)$ to be slowly varying and expand $H=H^{(0)}+H^{(1)}+\cdots$ in inverse powers of the dimensionless quantity $g r^{3}$. We will compute the effective Hamiltonian in perturbation theory by integrating out the other fields in their ground state, in which $(r, \theta, \phi)$ appear as parameters. Similar analysis to this was performed in [20-25]. Defining $\vec{\partial} \equiv\left(\partial_{\mathbf{x}_{1}}, \partial_{\mathbf{x}_{2}}\right)$, the Hamiltonian, to lowest order, is

$$
\begin{aligned}
H^{(0)} \equiv & -\frac{1}{2\left(\mathbf{x}_{1}^{2}-\mathbf{x}_{2}^{2}\right)} \vec{\partial} \cdot\left(\mathbf{x}_{1}^{2}-\mathbf{x}_{2}^{2}\right) \vec{\partial} \\
& -\frac{1}{2\left(\mathbf{x}_{1}^{2}-\mathbf{x}_{2}^{2}\right)^{2}}\left[\left(\mathbf{x}_{1}^{2}+\mathbf{x}_{2}^{2}\right)\left(\partial_{\vartheta_{3}}^{2}+\partial_{\varphi_{3}}^{2}\right)+4 \mathbf{x}_{1} \mathbf{x}_{2} \partial_{\vartheta_{3}} \partial_{\varphi_{3}}\right] \\
& +\frac{g^{2}}{2} r^{2}\left(\mathbf{x}_{1}^{2}+\mathbf{x}_{2}^{2}\right)-\operatorname{igr\epsilon }_{3 D E} \bar{\chi}_{D} \tilde{\boldsymbol{\sigma}}^{3} \chi_{E}
\end{aligned}
$$

where $\tilde{\boldsymbol{\sigma}}_{\alpha}^{i \beta} \equiv M^{j i} \boldsymbol{\sigma}_{\alpha}^{j \beta}$ depends explicitly on $\left(\vartheta_{1}, \vartheta_{2}, \vartheta_{3}\right)$. It is straightforward to show that $H^{(0)}$ admits a zero energy ground state given by

\footnotetext{
${ }^{3}$ Also sometimes called a Coulomb branch.
}

$$
\begin{aligned}
\Psi^{(0)}= & \frac{g r}{\pi \sqrt{32}} e^{-\frac{g}{2} r\left(\mathbf{x}_{1}^{2}+\mathbf{x}_{2}^{2}\right)} \\
& \times \sum_{B=1}^{2}\left\{\bar{\chi}_{B} \epsilon \bar{\chi}_{B}-i \sum_{C=1}^{2} \epsilon_{3 B C} \bar{\chi}_{B} \tilde{\sigma}^{3}\left(\bar{\chi}_{C} \epsilon\right)\right\}|0\rangle,
\end{aligned}
$$

where $|0\rangle$ is the fermionic vacuum and we have normalized $\Psi^{(0)}$ with respect to

$$
\int_{0}^{\infty} d \mathbf{x}_{1} \int_{-\mathbf{x}_{1}}^{\mathbf{x}_{1}} d \mathbf{x}_{2} \int_{0}^{2 \pi} d \vartheta_{3} \int_{0}^{2 \pi} d \varphi_{3}\left(\mathbf{x}_{1}^{2}-\mathbf{x}_{2}^{2}\right) .
$$

Similarly we can expand the supercharges $Q_{\alpha}=Q_{\alpha}^{(0)}+$ $Q_{\alpha}^{(1)}+\cdots$, where

$$
\begin{aligned}
Q_{\alpha}^{(0)} \equiv & -i \sum_{a, b=1}^{2} \tilde{\boldsymbol{\sigma}}_{\alpha}^{b \gamma} \chi_{a \gamma}\left(\delta_{a b}\left\{\partial_{\mathbf{x}_{b}}+\frac{g}{2}\left|\epsilon_{b s t}\right| \mathbf{x}_{s} \mathbf{x}_{t}\right\}\right. \\
& \left.+i \frac{\epsilon_{a b c}}{\mathbf{x}_{a}^{2}-\mathbf{x}_{b}^{2}}\left(\mathbf{x}_{a} \mathcal{P}^{c}+\mathbf{x}_{b} \mathcal{S}_{c}\right)\right) \\
Q_{\alpha}^{(1)} \equiv & -i \tilde{\boldsymbol{\sigma}}_{\alpha}^{b \gamma} \chi_{3 \gamma}\left(\delta_{3 b}\left\{\partial_{r}+\frac{g}{2}\left|\epsilon_{3 s t}\right| \mathbf{x}_{s} \mathbf{x}_{t}\right\}\right. \\
& \left.+i \frac{\epsilon_{3 b c}}{r}\left(\mathcal{P}^{c}+\mathcal{S}_{c}\right)\right) .
\end{aligned}
$$

It is easy to check that $Q_{\alpha}^{(0)} \Psi^{(0)}=\bar{Q}^{(0) \beta} \Psi^{(0)}=0$. We are now tasked with finding the effective supercharges $Q_{\alpha}^{\text {eff }}=$ $\left\langle Q_{\alpha}^{(1)}\right\rangle_{\Psi^{(0)}}+\cdots$ that act on the massless degrees of freedom $(r, \theta, \phi)$ along the moduli space. At lowest order we find the supercharges (acting on gauge-invariant wave functions) are those of a free particle in $\mathbb{R}^{3}$ and its fermionic superpartner,

$$
Q_{\alpha}^{\mathrm{eff}}=-i \nabla_{\overrightarrow{\mathbf{x}}} \cdot \overrightarrow{\boldsymbol{\sigma}}_{\alpha}^{\gamma} \psi_{\gamma}, \quad \bar{Q}_{\mathrm{eff}}^{\beta}=-i \bar{\psi}^{\gamma} \nabla_{\overrightarrow{\mathbf{x}}} \cdot \overrightarrow{\boldsymbol{\sigma}}_{\gamma}^{\beta},
$$

where we have labeled $(r, \theta, \phi)$ in Cartesian coordinates as well as defined $\left(\psi_{\alpha}, \bar{\psi}^{\beta}\right) \equiv\left(\chi_{3 \alpha}, \bar{\chi}_{3}^{\beta}\right)$. Since the remaining 
gauge angles $\left(\varphi_{1}, \varphi_{2}\right)$ have no kinetic terms in the effective theory along the moduli space, we need not consider them as dynamical variables and can treat $\psi_{\alpha}$ as a fundamental field.

Let us now compute the effective theory to next order in perturbation theory. Instead of computing this in the operator formalism, let us first invoke symmetry arguments to constrain what the answer should look like. The low energy effective theory on the moduli space should be a supersymmetric theory with four supercharges and an $S O$ (3) $R$ symmetry; therefore, it should fall in the class discovered in $[26,27]$,

$$
\begin{aligned}
\mathcal{L}= & \frac{1}{2} f\left(\dot{\overrightarrow{\mathbf{x}}}^{2}+i(\bar{\psi} \dot{\psi}-\dot{\bar{\psi}} \psi)+D^{2}\right)+\frac{1}{2}\left(\nabla_{k} f\right) \epsilon_{k l m} \dot{\mathbf{x}}^{l} \bar{\psi} \boldsymbol{\sigma}^{m} \psi \\
& -\frac{D}{2}\left(\nabla_{\overrightarrow{\mathbf{x}}} f\right) \cdot \bar{\psi} \overrightarrow{\boldsymbol{\sigma}} \psi+\frac{1}{4}\left(\nabla_{i} \nabla_{j} f\right)\left(\bar{\psi} \boldsymbol{\sigma}^{i} \psi\right)\left(\bar{\psi} \boldsymbol{\sigma}^{j} \psi\right),
\end{aligned}
$$

which is invariant under

$$
\begin{aligned}
\delta \overrightarrow{\mathbf{x}} & =i \bar{\psi} \overrightarrow{\boldsymbol{\sigma}} \xi-i \bar{\xi} \overrightarrow{\boldsymbol{\sigma}} \psi, \\
\delta \psi_{\alpha} & =\dot{\overrightarrow{\mathbf{x}}} \cdot \overrightarrow{\boldsymbol{\sigma}}_{\alpha}^{\beta} \xi_{\beta}+i D \xi_{\alpha}, \\
\delta \bar{\psi}^{\beta} & =\dot{\overrightarrow{\mathbf{x}}} \cdot \bar{\xi}^{\alpha} \overrightarrow{\boldsymbol{\sigma}}_{\alpha}^{\beta}-i D \bar{\xi}^{\beta}, \\
\delta D & =-\dot{\bar{\psi}} \xi-\bar{\xi} \dot{\psi} .
\end{aligned}
$$

To preserve the $S O(3)$ symmetry, $f$ should be a function of $r \equiv|\overrightarrow{\mathbf{x}}|$. Notice that (5.7) reduces to the theory of a free particle and its superpartner when $f=1$. Therefore we should find that at one-loop order $f=1+\frac{c}{g r^{3}}$, since $\left(g r^{3}\right)^{-1}$ is our expansion parameter, with $c$ to be determined. A calculation [22,23] reproduced in Appendix B gives $c=-3 / 2$ or

$$
f=1-\frac{3}{2 g r^{3}} .
$$

Analytic evidence for the numerically found supersymmetric ground states can be obtained by studying the Schrödinger problem associated with (5.7). We do not do this here, but we can gain some intuition by studying the existence of normalizable zero modes of the Laplacian on moduli space [28]:

$$
d s^{2}=\left(1-\frac{3}{2 g r^{3}}\right)\left(d r^{2}+r^{2} d \Omega_{2}^{2}\right) .
$$

We can construct two normalizable zero modes as follows. The zero form

$$
\omega_{0} \equiv \int^{r} d r^{\prime} \frac{1}{r^{\prime 2}}\left(1-\frac{3}{2 g r^{\prime 3}}\right)^{-1 / 2}
$$

is a zero mode of the Laplacian, but is not normalizable. To construct normalizable forms, we take

$$
\omega_{1} \equiv d \omega_{0}, \quad \omega_{2}=\star \omega_{1} .
$$

These are normalizable within the domain $r \in\left[\left(\frac{3}{2 g}\right)^{1 / 3}, \infty\right]$. Since there exists zero modes in this toy-moduli-space approximation, it would be interesting to study the set of ground states of (5.7) in more detail.

\section{DISCUSSION}

In this paper we have studied the mini-BFSS/BMN model with gauge group $S U(2)$ and uncovered numerical evidence for a set of supersymmetric ground states in the massless limit of the theory. In the massless limit the matrices can become widely separated. The effective theory on the moduli space has nontrivial interactions governed by a metric that gets generated on this moduli space at one loop.

Our numerical evidence for zero energy ground states is limited to the $N=2$ case, but we can now safely establish that the vanishing Witten index of mini-BFSS does not conclusively imply supersymmetry breaking, even for $N>2$. This should renew our interest in determining if supersymmetric states continue to exist at large $N$. Let us now discuss what may happen in the $S U(N)$ case at large $N$. The quartic interaction in (2.5) can be rewritten as a commutator-squared interaction $\left(f_{A B C} X_{B}^{i} X_{C}^{j}\right)^{2} \sim$ $\operatorname{Tr}\left(\left[X^{i}, X^{j}\right]\right)^{2}$, where $X^{i} \equiv X_{A}^{i} \tau_{A}$ and $\tau_{A}$ are the generators in the fundamental of $S U(N)$. Therefore, at tree level, along the moduli space there will be a set of $N-1$ massless, noninteracting, point particles in $\mathbb{R}^{3}$ (and their superpartners), each one corresponding to an element of the Cartan of $S U(N)$. At one loop there will be a correction to the moduli space metric, depending on the relative distances between these particles. Just as in the $S U(2)$ case these corrections will come at order $\left|r_{a}-r_{b}\right|^{-3}$. One difference, however, is that there may be an enhancement of order $N$ to this correction. It would certainly be interesting to see if we can isolate the $\left|r_{a}-r_{b}\right|^{-3}$ corrections to the moduli space metric by taking a large $N$ limit, as can be done in the D0-D4 system [28] and in the three-node Abelian quiver [29]. Perhaps we can adapt the methods in [30] for these purposes. The analysis in [22] seems to suggest that such a decoupling limit at large $N$ is possible.

Interestingly, it was shown in [29] that the one-loop effective action on the Coulomb branch of the three-node Abelian quiver exhibits an emergent conformal symmetry at large $N$. This conformal symmetry depends on the delicate balance between the form of the interaction potential and the metric on the moduli space, which has a similar $\left|r_{a}-r_{b}\right|^{-3}$ form as in (5.8). It would be interesting to establish whether the $S U(N)$ generalization of the model studied in this paper also has a nontrivial conformal symmetry at infinite $N$, broken by finite $N$ effects. We save this problem for future work, but list here some reasons why this would be worth studying: 
(1) The BFSS matrix model has a holographic interpretation [11,31-33]. At large $N$ it is dual to a background of D0 branes in type IIA supergravity. In BFSS there is no correction to the moduli space metric and neither side of this duality is conformal. The BFSS matrix model is thus a theory of the 10D flat space $S$ matrix. It would be interesting to understand the large $N$ version of mini-BFSS in the context of holography along similar lines. Because of the large number of coupled degrees of freedom at large $N$ and the reduced supersymmetry, the effective theory along the moduli space of mini-BFSS has a nontrivial metric and may potentially exhibit a nontrivial conformal fixed point along this moduli space, as happens for quiver quantum mechanics models with vector rather than matrix interactions [29,34]. To answer this question definitively we will need to compute the effective theory along the Coulomb branch for $N \gg 2$ and check whether it is conformal.

(2) New results have shown that a certain class of disordered quantum mechanics models, known as SYK for Sachdev-Ye-Kitaev, exhibits phenomenology of interest for near-extremal black holes (see [34-41] and references therein as well as [42-45] for models without disorder). These phenomena include an emergent conformal symmetry in the IR, maximal chaos [46], and a linear in $T$ specific heat. Despite the successes of these models, they are not dual to weakly coupled gravity. BFSS is a large $N$ gauged matrix quantum mechanics dual to weakly coupled Einstein gravity but, as we previously mentioned, it does not have an emergent conformal symmetry and remains a model of $D$ particles in flat space. It would certainly be interesting if mini-BFSS fell in the universality class of quantum mechanics models with emergent conformal symmetry in the IR and maximal chaos, such as the SYK model and its nondisordered cousins, but remains dual to weakly coupled gravity. Recently $[47,48]$ advocated the study of such matrix models for similar reasons. In the same vein the authors of [49] study classical chaos in BFSS numerically.

(3) If this model, like SYK, is at all related to the holography of near-extremal black holes, then we can try to study its $S$ matrix to gain some insight into the real time dynamics of black hole microstates. A numerical implementation of such a study in the context of similar supersymmetric quantum mechanics models with flat directions can be found in [50].

(4) The slow moving dynamics of a class of supersymmetric multicentered black hole solutions in supergravity is a superconformal quantum mechanics [51-54] with no potential, provided a near horizon limit is taken. It would be interesting to understand if there is some limit in which the multicentered black hole moduli space quantum mechanics and the large $N$ matrix quantum mechanics on the moduli space coincide, perhaps as a consequence of nonrenormalization theorems as in [2].

\section{ACKNOWLEDGMENTS}

It is a pleasure to thank Dionysios Anninos, Frederik Denef, Felix Haehl, Rachel Hathaway, Eliot Hijano, Jaehoon Lee, Eric Mintun, Edgar Shaghoulian, Benson Way, and Mark Van Raamsdonk for helpful discussions. We are particularly indebted to Dionysios Anninos, Frederik Denef, and Edgar Shaghoulian for their comments on an early draft. We made heavy use of Matthew Headrick's grassmann.m package. C. C. thanks David and Gay Cogburn for their support. T. A. is supported in part by the U.S. Department of Energy under Contract No. DE-SC0012567, by the Natural Sciences and Engineering Research Council of Canada, and by Grant No. 376206 from the Simons Foundation.

\section{APPENDIX A: REDUCED SCHRÖDINGER EQUATION}

In this Appendix we construct gauge-invariant highestweight wave functions of $S O(3)_{J}$ in each $R$-charge sector (up to 3) and use these to maximally reduce the Schrödinger equation via symmetries.

$$
\text { 1. } R=0
$$

This sector of the theory was studied in [55-57], although without access to numerics. We repeat their analysis here. We wish to separate variables using the $S O(3)_{J}$ symmetry. We therefore want to write down the highest weight state satisfying $J^{3}|\psi\rangle_{0}=j|\psi\rangle_{0}$ and $J^{+}|\psi\rangle_{0}=0$, with $J^{ \pm} \equiv J^{1} \pm i J^{2}$. The rest of the spin multiplet can be obtained by acting on $|\psi\rangle_{0}$ with $J^{-}$up to $2 j$ times. This, however, does not entirely fix the angular dependence of the wave function, as these two conditions only fix the dependence on up to two angles. Recall, however, that the operators $\vec{P}$ commute with $\vec{J}$ and $\vec{P}^{2}=\vec{J}^{2}$, but $[H, \vec{P}] \neq 0$. We will then write $|\psi\rangle_{0}$ as a sum of terms with definite $P^{3}$ eigenvalue. That is, we write $|\psi\rangle_{0}$ as

$$
|\psi\rangle_{0}=e^{i j \vartheta_{1}} \sin ^{j} \vartheta_{2} \sum_{p=-j}^{j} e^{i p \vartheta_{3}} \cot ^{p}\left(\frac{\vartheta_{2}}{2}\right) f^{p}\left(\mathbf{x}_{a}\right) .
$$

Since the number of terms in the wave function grows with $j$, it will be cumbersome to give the reduced radial Schrödinger equation for arbitrary $j$. Instead, we will give the expressions for $j=0, \frac{1}{2}, 1$. Before giving the reduced Schrödinger equations, it is worth noting that it has long been known that there exist no supersymmetric states in this sector [1]. The reason is that the supersymmetry equations 
$Q_{\alpha}|\psi\rangle_{0}=\bar{Q}^{\beta}|\psi\rangle_{0}=0$ are easy to solve and give

$$
|\psi\rangle_{0}^{\mathrm{SUSY}} \sim \exp \left\{g \mathbf{x}_{1} \mathbf{x}_{2} \mathbf{x}_{3}+\frac{m}{2} \mathbf{x}_{a} \mathbf{x}_{a}\right\}
$$

which is non-normalizable. It is also known that the spectrum in this sector is discrete [57].

For parsimony let us define

$$
\hat{\mathcal{H}} \equiv-\frac{1}{2 \Delta} \partial_{\mathbf{x}_{a}} \Delta \partial_{\mathbf{x}_{a}}+V
$$

with $V$ defined in (3.19). Then for $j=0$ the reduced Schrödinger equation, obtained from $H_{m}|\psi\rangle_{0}=\mathcal{E}_{m}|\psi\rangle_{0}$, is simply

$$
\left(\hat{\mathcal{H}}+\frac{9}{2} m\right) f^{0}\left(\mathbf{x}_{a}\right)=\mathcal{E}_{m} f^{0}\left(\mathbf{x}_{a}\right) .
$$

For $j=1 / 2$ there is no mixing between the $f^{ \pm 1 / 2}\left(\mathbf{x}_{a}\right)$, and each satisfies

$$
\left(\hat{\mathcal{H}}+\frac{9}{2} m+\frac{T}{8}\right) f^{ \pm 1 / 2}\left(\mathbf{x}_{a}\right)=\mathcal{E}_{m} f^{ \pm 1 / 2}\left(\mathbf{x}_{a}\right),
$$

where $T$ was defined in (3.22). Finally, for $j=1$ we have

$$
\begin{aligned}
\{\hat{\mathcal{H}} & \left.+\frac{9}{2} m+\frac{T}{4}+\frac{1}{4}\left(\begin{array}{ccc}
\mathbf{y}_{3} & 0 & \mathbf{y}_{1}-\mathbf{y}_{2} \\
0 & T-2 \mathbf{y}_{3} & 0 \\
\mathbf{y}_{1}-\mathbf{y}_{2} & 0 & \mathbf{y}_{3}
\end{array}\right)\right\} \\
\times\left(\begin{array}{c}
f^{-1} \\
f^{0} \\
f^{+1}
\end{array}\right) & =\mathcal{E}_{m}\left(\begin{array}{c}
f^{-1} \\
f^{0} \\
f^{+1}
\end{array}\right) .
\end{aligned}
$$

\section{2. $R=1$}

Continuing on from the last section, we want to write down wave functions in the $R=1$ sector that are gauge invariant, and satisfy $J^{3}|\psi\rangle_{1}=j|\psi\rangle_{1}$ and $J^{+}|\psi\rangle_{1}=0$. To do so, we will write our wave functions as

$$
|\psi\rangle_{1}=e^{i j \vartheta_{1}} \sin ^{j} \vartheta_{2} \sum_{p=-j}^{j} e^{i p \vartheta_{3}} \cot ^{p}\left(\frac{\vartheta_{2}}{2}\right) f_{A \alpha}^{p} \bar{\chi}_{A}^{\alpha}|0\rangle,
$$

where $|0\rangle$ is the fermionic vacuum and each term in the sum has a definite $P^{3}$ eigenvalue. The functions $f_{A \alpha}^{p}$ that satisfy these conditions are

$$
\begin{aligned}
f_{A 1}^{p}= & e^{-i \frac{\vartheta_{1}}{2}}\left\{e^{-i \frac{\vartheta_{3}}{2}} \cos \left(\frac{\vartheta_{2}}{2}\right) L_{2 A-1}^{p}\left(\mathbf{x}_{a}\right)\right. \\
& \left.-e^{i \frac{\vartheta_{3}}{2}} \sin \left(\frac{\vartheta_{2}}{2}\right) L_{2 A}^{p}\left(\mathbf{x}_{a}\right)\right\}, \\
f_{A 2}^{p}= & e^{i \frac{\vartheta_{1}}{2}}\left\{e^{-i \frac{\vartheta_{3}}{2}} \sin \left(\frac{\vartheta_{2}}{2}\right) L_{2 A-1}^{p}\left(\mathbf{x}_{a}\right)\right. \\
& \left.+e^{i \frac{\vartheta_{3}}{2}} \cos \left(\frac{\vartheta_{2}}{2}\right) L_{2 A}^{p}\left(\mathbf{x}_{a}\right)\right\} .
\end{aligned}
$$

We remind the reader that the $\bar{\chi}_{A}^{\alpha}$ are the gauge-invariant fermions defined in (3.23). The reduced Schrödinger equation for $j=0$ (and hence $p=0$ ) is

$$
\left\{\hat{\mathcal{H}}+\frac{7}{2} m+\frac{5}{8} T+\mathbf{A}\right\}\left(\begin{array}{c}
L_{1}^{0} \\
\vdots \\
L_{6}^{0}
\end{array}\right)=\mathcal{E}_{m}\left(\begin{array}{c}
L_{1}^{0} \\
\vdots \\
L_{6}^{0}
\end{array}\right),
$$

where $\mathbf{A}$ is a $6 \times 6$ matrix that can be written in terms of $2 \times 2$ blocks as follows:

$\mathbf{A} \equiv \frac{i}{2}\left(\begin{array}{ccc}i \mathbf{y}_{1} \mathbb{1} & -\left(2 g \mathbf{x}_{3}+\mathbf{z}_{3}\right) \boldsymbol{\sigma}^{3} & \left(2 g \mathbf{x}_{2}+\mathbf{z}_{2}\right) \boldsymbol{\sigma}^{2} \\ \left(2 g \mathbf{x}_{3}+\mathbf{z}_{3}\right) \boldsymbol{\sigma}^{3} & i \mathbf{y}_{2} \mathbb{1} & -\left(2 g \mathbf{x}_{1}+\mathbf{z}_{1}\right) \boldsymbol{\sigma}^{1} \\ -\left(2 g \mathbf{x}_{2}+\mathbf{z}_{2}\right) \boldsymbol{\sigma}^{2} & \left(2 g \mathbf{x}_{1}+\mathbf{z}_{1}\right) \boldsymbol{\sigma}^{1} & i \mathbf{y}_{3} \mathbb{1}\end{array}\right)$,

where the coordinates $\mathbf{y}_{a}$ and $\mathbf{z}_{a}$ (nonlinearly related to $\mathbf{x}_{a}$ ) were defined in (3.20).

Using the above definitions it is straightforward to write down the equations for $j=1 / 2$. These are

$$
\begin{aligned}
& \left\{\hat{\mathcal{H}}+\frac{7}{2} m+\frac{3}{4} T+\left(\begin{array}{c|c}
\mathbf{A}+\mathbf{B} & \mathbf{C} \\
\hline \mathbf{C}^{\dagger} & \mathbf{A}-\mathbf{B}
\end{array}\right)\right\} \\
& \times\left(\begin{array}{c}
L_{1}^{-\frac{1}{2}} \\
\vdots \\
L_{6}^{-\frac{1}{2}} \\
L_{1}^{\frac{1}{2}} \\
\vdots \\
L_{6}^{\frac{1}{2}}
\end{array}\right)=\mathcal{E}_{m}\left(\begin{array}{c}
L_{1}^{-\frac{1}{2}} \\
\vdots \\
L_{6}^{-\frac{1}{2}} \\
L_{1}^{\frac{1}{2}} \\
\vdots \\
L_{6}^{\frac{1}{2}}
\end{array}\right)
\end{aligned}
$$

with

$$
\mathbf{B} \equiv \frac{1}{4}\left(\begin{array}{ccc}
\mathbf{y}_{3} \boldsymbol{\sigma}^{3} & -2 i \mathbf{z}_{3} \mathbb{1} & 0 \\
2 i \mathbf{z}_{3} \mathbb{1} & \mathbf{y}_{3} \boldsymbol{\sigma}^{3} & 0 \\
0 & 0 & \mathbf{y}_{3} \boldsymbol{\sigma}^{3}
\end{array}\right)
$$


and

$$
\mathbf{C} \equiv \frac{1}{4}\left(\begin{array}{ccc}
\mathbf{y}_{1} \boldsymbol{\sigma}^{1}-i \mathbf{y}_{2} \boldsymbol{\sigma}^{2} & 0 & 2 \mathbf{z}_{2} \mathbb{1} \\
0 & \mathbf{y}_{1} \boldsymbol{\sigma}^{1}-i \mathbf{y}_{2} \boldsymbol{\sigma}^{2} & -2 i \mathbf{z}_{1} \mathbb{1} \\
-2 \mathbf{z}_{2} \mathbb{1} & 2 i \mathbf{z}_{1} \mathbb{1} & \mathbf{y}_{1} \boldsymbol{\sigma}^{1}-i \mathbf{y}_{2} \boldsymbol{\sigma}^{2}
\end{array}\right) .
$$

\section{3. $R=2$}

As we can see, the number of equations keeps increasing with fermion number and spin. Therefore in this section and the next, we will only give the reduced Schrödinger equations for $j=0$. As before the general highest weight $R=2$ wave function admits a decomposition:

$$
|\psi\rangle_{2}=e^{i j \vartheta_{1}} \sin ^{j} \vartheta_{2} \sum_{p=-j}^{j} e^{i p \vartheta_{3}} \cot ^{p}\left(\frac{\vartheta_{2}}{2}\right) f_{A B \alpha \beta}^{p} \bar{\chi}_{A}^{\alpha} \bar{\chi}_{B}^{\beta}|0\rangle .
$$

To avoid overcounting let us set $f_{A B \alpha \beta}^{p}=0$ whenever $B<A$ and similarly $f_{A A \alpha \beta}^{p}=0$ (no sum on indices) whenever $\beta \leq \alpha$. Imposing that $J^{3}|\psi\rangle_{2}=j|\psi\rangle_{2}, J^{+}|\psi\rangle_{2}=$ 0 and that each term in the sum has definite $P^{3}$ eigenvalue imposes that the functions $f_{A B \alpha \beta}^{p}$ take on a particular form. These are (no sum on indices and $A<B$ )

$$
\begin{gathered}
f_{A A 12}^{p}=L_{A}^{p}\left(\mathbf{x}_{a}\right), \\
f_{A B 12}^{p}=\frac{e^{-i \vartheta_{3}}}{2} \sin \vartheta_{2} Y_{A B}^{p}\left(\mathbf{x}_{a}\right)+\cos ^{2}\left(\frac{\vartheta_{2}}{2}\right) R_{A B}^{p}\left(\mathbf{x}_{a}\right)-\sin ^{2}\left(\frac{\vartheta_{2}}{2}\right) S_{A B}^{p}\left(\mathbf{x}_{a}\right)-\frac{e^{i \vartheta_{3}}}{2} \sin \vartheta_{2} U_{A B}^{p}\left(\mathbf{x}_{a}\right), \\
f_{A B 21}^{p}=\frac{e^{-i \vartheta_{3}}}{2} \sin \vartheta_{2} Y_{A B}^{p}\left(\mathbf{x}_{a}\right)-\sin ^{2}\left(\frac{\vartheta_{2}}{2}\right) R_{A B}^{p}\left(\mathbf{x}_{a}\right)+\cos ^{2}\left(\frac{\vartheta_{2}}{2}\right) S_{A B}^{p}\left(\mathbf{x}_{a}\right)-\frac{e^{i \vartheta_{3}}}{2} \sin \vartheta_{2} U_{A B}^{p}\left(\mathbf{x}_{a}\right), \\
f_{A B 11}^{p}=e^{-i \vartheta_{1}}\left\{e^{-i \vartheta_{3}} \cos ^{2}\left(\frac{\vartheta_{2}}{2}\right) Y_{A B}^{p}\left(\mathbf{x}_{a}\right)-\frac{1}{2} \sin \vartheta_{2}\left(R_{A B}^{p}\left(\mathbf{x}_{a}\right)+S_{A B}^{p}\left(\mathbf{x}_{a}\right)\right)+e^{i \vartheta_{3}} \sin ^{2}\left(\frac{\vartheta_{2}}{2}\right) U_{A B}^{p}\left(\mathbf{x}_{a}\right)\right\}, \\
f_{A B 22}^{p}=e^{i \vartheta_{1}}\left\{e^{-i \vartheta_{3}} \sin ^{2}\left(\frac{\vartheta_{2}}{2}\right) Y_{A B}^{p}\left(\mathbf{x}_{a}\right)+\frac{1}{2} \sin \vartheta_{2}\left(R_{A B}^{p}\left(\mathbf{x}_{a}\right)+S_{A B}^{p}\left(\mathbf{x}_{a}\right)\right)+e^{i \vartheta_{3}} \cos ^{2}\left(\frac{\vartheta_{2}}{2}\right) U_{A B}^{p}\left(\mathbf{x}_{a}\right)\right\}
\end{gathered}
$$

Notice that even for $j=0$, determining the spectrum will involve solving a set of 15 coupled partial differential equations. We will label the set of functions $Y_{A B}^{p} \equiv Y_{6-A-B}^{p}$ and so on for the remaining functions. We also define the following vector of functions:

$$
\Psi_{R=2}^{0} \equiv\left(L_{1}^{0}, \ldots, R_{1}^{0}, \ldots, S_{1}^{0}, \ldots, U_{1}^{0}, \ldots, Y_{1}^{0}, \ldots\right)^{\mathrm{T}} .
$$

The $j=0$ Schrödinger equation is then

$$
\left\{\hat{\mathcal{H}}+\frac{5}{2} m+\frac{3}{4} T+\mathbf{D}+\mathbf{L}+g \mathbf{M}\right\} \Psi_{R=2}^{0}=\mathcal{E}_{m} \Psi_{R=2}^{0},
$$

where $\mathbf{D}, \mathbf{L}$, and $\mathbf{M}$ are $15 \times 15$ matrices that can be written in terms of $3 \times 3$ blocks as follows:

$$
\mathbf{D} \equiv\left(\begin{array}{ccccc}
\mathbf{d}^{1} & 0 & 0 & 0 & 0 \\
0 & \mathbf{d}^{3} & \mathbf{d}^{1}-\frac{\mathbf{y}_{3}}{4} \mathbb{1} & 0 & 0 \\
0 & \mathbf{d}^{1}-\frac{\mathbf{y}_{3} \mathbb{4}}{4} & \mathbf{d}^{3} & 0 & 0 \\
0 & 0 & 0 & -\mathbf{d}^{3} & \frac{1}{4}\left(\mathbf{y}_{1}-\mathbf{y}_{2}\right) \mathbb{1} \\
0 & 0 & 0 & \frac{1}{4}\left(\mathbf{y}_{1}-\mathbf{y}_{2}\right) \mathbb{1} & -\mathbf{d}^{3}
\end{array}\right),
$$




$$
\begin{aligned}
& \mathbf{L} \equiv-\frac{1}{2}\left(\begin{array}{ccccc}
2 \sum_{a} \mathbf{y}_{a}\left|\mathcal{L}^{a}\right| & 0 & 0 & 0 & 0 \\
0 & 0 & 0 & \mathbf{z}_{1} \mathcal{L}^{1}+i \mathbf{z}_{2} \mathcal{L}^{2} & \mathbf{z}_{1} \mathcal{L}^{1}-i \mathbf{z}_{2} \mathcal{L}^{2} \\
0 & 0 & 0 & \mathbf{z}_{1} \mathcal{L}^{1}+i \mathbf{z}_{2} \mathcal{L}^{2} & \mathbf{z}_{1} \mathcal{L}^{1}-i \mathbf{z}_{2} \mathcal{L}^{2} \\
0 & \mathbf{z}_{1} \mathcal{L}^{1}-i \mathbf{z}_{2} \mathcal{L}^{2} & \mathbf{z}_{1} \mathcal{L}^{1}-i \mathbf{z}_{2} \mathcal{L}^{2} & -2 \mathbf{z}_{3} \mathcal{L}^{3} & 0 \\
0 & \mathbf{z}_{1} \mathcal{L}^{1}+i \mathbf{z}_{2} \mathcal{L}^{2} & \mathbf{z}_{1} \mathcal{L}^{1}+i \mathbf{z}_{2} \mathcal{L}^{2} & 0 & 2 \mathbf{z}_{3} \mathcal{L}^{3}
\end{array}\right) \\
& \mathbf{M} \equiv\left(\begin{array}{ccccc}
0 & \mathbf{x}_{3} \mathbf{m}^{3} & \mathbf{x}_{3} \mathbf{m}^{3} & \mathbf{x}_{1} \mathbf{m}^{1}+\mathbf{x}_{2} \mathbf{m}^{2} & \mathbf{x}_{2} \mathbf{m}^{2}-\mathbf{x}_{1} \mathbf{m}^{1} \\
\mathbf{x}_{3} \mathbf{m}^{3 \dagger} & -\mathbf{x}_{3} \mathcal{L}^{3} & 0 & \mathbf{x}_{2} \mathbf{d}^{2} & \mathbf{x}_{2} \mathbf{d}^{2 \dagger}-\mathbf{x}_{1} \mathcal{L}^{1} \\
\mathbf{x}_{3} \mathbf{m}^{3 \dagger} & 0 & \mathbf{x}_{3} \mathcal{L}^{3} & -\mathbf{x}_{1} \mathcal{L}^{1}-\mathbf{x}_{2} \mathbf{d}^{2 \dagger} & -\mathbf{x}_{2} \mathbf{d}^{2} \\
\mathbf{x}_{1} \mathbf{m}^{1 \dagger}+\mathbf{x}_{2} \mathbf{m}^{2 \dagger} & \mathbf{x}_{2} \mathbf{d}^{2 \dagger} & -\mathbf{x}_{1} \mathcal{L}^{1}-\mathbf{x}_{2} \mathbf{d}^{2} & \mathbf{x}_{3} \mathcal{L}^{3} & 0 \\
\mathbf{x}_{2} \mathbf{m}^{2 \dagger}-\mathbf{x}_{1} \mathbf{m}^{1 \dagger} & \mathbf{x}_{2} \mathbf{d}^{2}-\mathbf{x}_{1} \mathcal{L}^{1} & -\mathbf{x}_{2} \mathbf{d}^{2 \dagger} & 0 & -\mathbf{x}_{3} \mathcal{L}^{3}
\end{array}\right) .
\end{aligned}
$$

In these definitions, the $\mathcal{L}^{i}$ are the $3 \times 3$ generators of $S O$ (3) defined below (3.1). The $\mathbf{d}^{i}$ are

$$
\begin{aligned}
\mathbf{d}^{1} & \equiv\left(\frac{T}{4}-\mathbf{y}_{a}\right) \delta_{a b}, \quad \mathbf{d}^{2} \equiv\left(\begin{array}{ccc}
0 & 0 & 0 \\
0 & 0 & 0 \\
-1 & 0 & 0
\end{array}\right), \\
\mathbf{d}^{3} & \equiv \frac{1}{2}\left(\mathbf{y}_{a}-\frac{1}{2} \mathbf{y}_{3}\right) \delta_{a b},
\end{aligned}
$$

and the $\mathbf{m}^{i}$ are

$$
\begin{aligned}
\mathbf{m}^{1} \equiv\left(\begin{array}{ccc}
0 & 0 & 0 \\
i & 0 & 0 \\
i & 0 & 0
\end{array}\right), \quad \mathbf{m}^{2} \equiv\left(\begin{array}{ccc}
0 & -1 & 0 \\
0 & 0 & 0 \\
0 & -1 & 0
\end{array}\right) \\
\mathbf{m}^{3} \equiv\left(\begin{array}{lll}
0 & 0 & i \\
0 & 0 & i \\
0 & 0 & 0
\end{array}\right)
\end{aligned}
$$

Whenever a matrix appears in an absolute value symbol $|\cdot|$, the absolute value is to be applied to the entries of the matrix.

The Schrödinger operator for $j=1 / 2$ will be a generalization of the above operator to one acting on 30 functions. We do not provide expressions for it here, but analyze its spectrum in the main text.

\section{4. $R=3$}

The highest weight $R=3$ wave functions take the form $|\psi\rangle_{3}=e^{i j \vartheta_{1}} \sin ^{j} \vartheta_{2} \sum_{p=-j}^{j} e^{i p \vartheta_{3}} \cot ^{p}\left(\frac{\vartheta_{2}}{2}\right) f_{A B C \alpha \beta \gamma}^{p} \bar{\chi}_{A}^{\alpha} \bar{\chi}_{B}^{\beta} \bar{\chi}_{C}^{\gamma}|0\rangle$.

To avoid overcounting we set

$$
f_{A B C \alpha \beta \gamma}^{p}=0 \quad \text { if } C<B \quad \text { or } \quad B<A,
$$

$$
\begin{array}{ll}
f_{A A B \alpha \beta \gamma}^{p}=0 & \text { if } \beta \leq \alpha, \\
f_{A B B \alpha \beta \gamma}^{p}=0 & \text { if } \gamma \leq \beta .
\end{array}
$$

Because of the fermionic statistics $f_{A A A \alpha \beta \gamma}^{p}=0$ identically. Imposing the highest weight condition forces $f_{123 \alpha \beta \gamma}^{p}$ to take the following form:

$$
f_{123 \alpha \beta \gamma}^{p}=\sum_{a, b, c=1}^{2} F_{a b c}^{p}\left(\mathbf{x}_{a}\right) u_{\alpha a}(\vec{\vartheta}) u_{\beta b}(\vec{\vartheta}) u_{\gamma c}(\vec{\vartheta}),
$$

with

$$
\begin{aligned}
u_{\alpha a}(\vec{\vartheta}) \equiv & e^{\frac{i}{2}\left((-1)^{\alpha} \vartheta_{1}+(-1)^{a} \vartheta_{3}\right)}\left\{(1-|\alpha-a|) \cos \left(\frac{\vartheta_{2}}{2}\right)\right. \\
& \left.+(\alpha-a) \sin \left(\frac{\vartheta_{2}}{2}\right)\right\} .
\end{aligned}
$$

Furthermore

$f_{A A B \alpha \beta \gamma}^{p}=U_{A A B}^{p}\left(\mathbf{x}_{a}\right) y_{\alpha \beta \gamma}^{1}(\vec{\vartheta})+Y_{A A B}^{p}\left(\mathbf{x}_{a}\right) y_{\alpha \beta \gamma}^{2}(\vec{\vartheta})$,

$f_{A B B \alpha \beta \gamma}^{p}=U_{A B B}^{p}\left(\mathbf{x}_{a}\right) y_{\alpha \beta \gamma}^{1}(\vec{\vartheta})+Y_{A B B}^{p}\left(\mathbf{x}_{a}\right) y_{\alpha \beta \gamma}^{2}(\vec{\vartheta})$,

where

$$
\begin{aligned}
y_{\alpha \beta \gamma}^{1}(\vec{\vartheta}) \equiv & \frac{e^{\frac{i}{2}\left\{\left((-1)^{\alpha}+(-1)^{\beta}+(-1)^{\gamma}\right) \vartheta_{1}-\vartheta_{3}\right\}}}{2} \\
& \times\left[(4-\alpha \beta \gamma) \cos \left(\frac{\vartheta_{2}}{2}\right)+(\alpha \beta \gamma-2) \sin \left(\frac{\vartheta_{2}}{2}\right)\right], \\
y_{\alpha \beta \gamma}^{2}(\vec{\vartheta}) \equiv & \frac{e^{\frac{i}{2}\left\{\left((-1)^{\alpha}+(-1)^{\beta}+(-1)^{\gamma}\right) \vartheta_{1}+\vartheta_{3}\right\}}}{2} \\
& \times\left[(\alpha \beta \gamma-4) \sin \left(\frac{\vartheta_{2}}{2}\right)+(\alpha \beta \gamma-2) \cos \left(\frac{\vartheta_{2}}{2}\right)\right] .
\end{aligned}
$$


Notice that for $j=0$ the reduced Schrödinger equation is a set of 20 coupled partial differential equations. We will give the Schrödinger operator acting on the following vector of functions:

$$
\begin{aligned}
\Psi_{R=3}^{0} \equiv & \left(F_{122}^{0}, F_{211}^{0}, F_{121}^{0}, F_{212}^{0}, F_{221}^{0}, F_{112}^{0}, F_{111}^{0}, F_{222}^{0},\right. \\
& U_{113}^{0}, U_{223}^{0}, Y_{113}^{0}, Y_{223}^{0}, U_{112}^{0}, U_{233}^{0}, Y_{112}^{0}, Y_{233}^{0}, \\
& \left.U_{122}^{0}, U_{133}^{0} Y_{122}^{0}, Y_{133}^{0}\right)^{\mathrm{T}} .
\end{aligned}
$$

With $\Psi_{R=3}^{0}$ defined, we are tasked with solving the following set of differential equations:

$$
\begin{aligned}
& \left\{\hat{\mathcal{H}}+\frac{3}{2} m+\frac{1}{4}\left(\frac{11}{2} T-\mathbf{y}_{3}\right)-\mathbf{I}+\mathbf{J}+\mathbf{J}^{\dagger}+\mathbf{K}\right\} \Psi_{R=3}^{0} \\
& =\mathcal{E}_{m} \Psi_{R=3}^{0},
\end{aligned}
$$

where $\mathbf{I}, \mathbf{J}$, and $\mathbf{K}$ are $20 \times 20$ matrices that can be written in block form as follows:

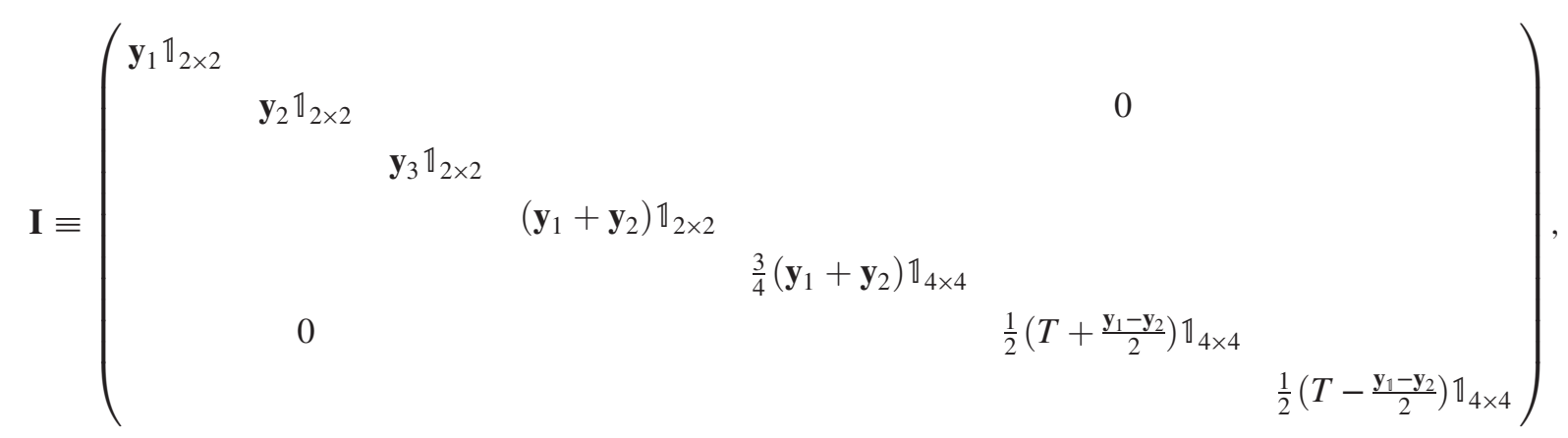

and $\mathbf{J}$ and $\mathbf{K}$ can be written in terms of $4 \times 4$ blocks as follows:

$$
\begin{aligned}
& \mathbf{J}=\frac{i}{2}\left(\begin{array}{ccccc}
0 & 0 & \mathbf{z}_{3} \mathbf{a}^{3}+2 g \mathbf{x}_{3} \mathbf{b}^{3} & \mathbf{z}_{2} \mathbf{a}^{2}+2 g \mathbf{x}_{2} \mathbf{b}^{2} & \mathbf{z}_{1} \mathbf{a}^{1}+2 g \mathbf{x}_{1} \mathbf{b}^{1} \\
0 & 0 & 0 & -\mathbf{z}_{2} \mathbf{a}^{2}+2 g \mathbf{x}_{2} \mathbf{c}^{2} & \mathbf{z}_{1} \mathbf{e}^{1}+2 g \mathbf{x}_{1} \mathbf{c}^{1} \\
0 & 0 & 0 & \boldsymbol{\sigma}^{1} \otimes\left(\mathbf{z}_{1} \mathbb{1}+2 g \mathbf{x}_{1} \boldsymbol{\sigma}^{3}\right) & -\boldsymbol{\sigma}^{2} \otimes\left(\mathbf{z}_{2} \boldsymbol{\sigma}^{1}-2 i g \mathbf{x}_{2} \boldsymbol{\sigma}^{2}\right) \\
0 & 0 & 0 & 0 & \boldsymbol{\sigma}^{3} \otimes\left(\mathbf{z}_{3} \mathbb{1}-2 g \mathbf{x}_{3} \boldsymbol{\sigma}^{3}\right) \\
0 & 0 & 0 & 0 & 0
\end{array}\right), \\
& \mathbf{K} \equiv\left(\begin{array}{ccccc}
\frac{1}{4}\left(T-5 \mathbf{y}_{3}\right) \boldsymbol{\sigma}^{1} \otimes \boldsymbol{\sigma}^{1} & \frac{1}{4}\left(\mathbf{y}_{1} \mathbf{s}+\mathbf{y}_{2} \mathbf{t}\right) & 0 & 0 & 0 \\
\frac{1}{4}\left(\mathbf{y}_{1} \mathbf{s}+\mathbf{y}_{2} \mathbf{t}\right)^{\dagger} & \frac{1}{4}\left(\mathbf{y}_{1}-\mathbf{y}_{2}\right) \boldsymbol{\sigma}^{1} \otimes \mathbb{1} & 0 & 0 & 0 \\
0 & 0 & -\mathbf{y}_{3} \mathbb{1} \otimes \boldsymbol{\sigma}^{1} & 0 & 0 \\
0 & 0 & 0 & -\mathbf{y}_{2} \mathbb{1} \otimes \boldsymbol{\sigma}^{1} & 0 \\
0 & 0 & 0 & 0 & -\mathbf{y}_{1} \mathbb{1} \otimes \boldsymbol{\sigma}^{1}
\end{array}\right),
\end{aligned}
$$

where we have implicitly defined

$$
\begin{aligned}
& \mathbf{a}^{1} \equiv-\frac{1}{2}\left(\begin{array}{ll}
0 & 0 \\
1 & 1
\end{array}\right) \otimes\left(\mathbb{1}-\boldsymbol{\sigma}^{1}\right)+\frac{1}{2}\left(\begin{array}{cc}
0 & 0 \\
1 & -1
\end{array}\right) \otimes\left(-i \boldsymbol{\sigma}^{2}+\boldsymbol{\sigma}^{3}\right), \quad \mathbf{b}^{1} \equiv \frac{1}{2}\left(\begin{array}{cc}
-1 & 1 \\
0 & 0
\end{array}\right) \otimes\left(\mathbb{1}+\boldsymbol{\sigma}^{1}\right)-\frac{1}{2}\left(\begin{array}{ll}
1 & 1 \\
0 & 0
\end{array}\right) \otimes\left(i \boldsymbol{\sigma}^{2}+\boldsymbol{\sigma}^{3}\right), \\
& \mathbf{a}^{2} \equiv-\frac{i}{2}\left(\begin{array}{cc}
-1 & 1 \\
0 & 0
\end{array}\right) \otimes\left(\mathbb{1}-\boldsymbol{\sigma}^{1}\right)+\frac{i}{2}\left(\begin{array}{ll}
1 & 1 \\
0 & 0
\end{array}\right) \otimes\left(-i \boldsymbol{\sigma}^{2}+\boldsymbol{\sigma}^{3}\right), \quad \mathbf{b}^{2} \equiv-\frac{i}{2}\left(\begin{array}{ll}
0 & 0 \\
1 & 1
\end{array}\right) \otimes\left(\mathbb{1}+\boldsymbol{\sigma}^{1}\right)+\frac{i}{2}\left(\begin{array}{cc}
0 & 0 \\
1 & -1
\end{array}\right) \otimes\left(i \boldsymbol{\sigma}^{2}+\boldsymbol{\sigma}^{3}\right), \\
& \mathbf{a}^{3} \equiv i \boldsymbol{\sigma}^{2} \otimes\left(\begin{array}{cc}
-1 & 1 \\
0 & 0
\end{array}\right)+\boldsymbol{\sigma}^{3} \otimes\left(\begin{array}{cc}
0 & 0 \\
-1 & 1
\end{array}\right), \quad \mathbf{b}^{3} \equiv-\mathbb{1} \otimes\left(\begin{array}{ll}
0 & 0 \\
1 & 1
\end{array}\right)-\boldsymbol{\sigma}^{1} \otimes\left(\begin{array}{ll}
1 & 1 \\
0 & 0
\end{array}\right),
\end{aligned}
$$

as well as 


$$
\begin{aligned}
& \mathbf{c}^{1} \equiv \frac{1}{2}\left(\begin{array}{cc}
0 & 0 \\
1 & -1
\end{array}\right) \otimes\left(\mathbb{1}+\boldsymbol{\sigma}^{1}\right)+\frac{1}{2}\left(\begin{array}{cc}
0 & 0 \\
1 & 1
\end{array}\right) \otimes\left(i \boldsymbol{\sigma}^{2}+\boldsymbol{\sigma}^{3}\right), \\
& \mathbf{c}^{2} \equiv-\frac{i}{2}\left(\begin{array}{ll}
0 & 0 \\
1 & 1
\end{array}\right) \otimes\left(1+\boldsymbol{\sigma}^{1}\right)-\frac{i}{2}\left(\begin{array}{cc}
0 & 0 \\
1 & -1
\end{array}\right) \otimes\left(i \boldsymbol{\sigma}^{2}+\boldsymbol{\sigma}^{3}\right), \\
& \mathbf{e}^{1} \equiv-\frac{1}{2}\left(\begin{array}{ll}
1 & 1 \\
0 & 0
\end{array}\right) \otimes\left(\mathbb{1}-\boldsymbol{\sigma}^{1}\right)+\frac{1}{2}\left(\begin{array}{cc}
-1 & 1 \\
0 & 0
\end{array}\right) \otimes\left(-i \boldsymbol{\sigma}^{2}+\boldsymbol{\sigma}^{3}\right),
\end{aligned}
$$

and finally

$$
\mathbf{s} \equiv\left(\begin{array}{ll}
1 & 1 \\
0 & 0
\end{array}\right) \otimes \mathbb{1}+\left(\begin{array}{cc}
0 & 0 \\
-3 & 1
\end{array}\right) \otimes \boldsymbol{\sigma}^{1} \quad \text { and } \quad \mathbf{t} \equiv\left(\begin{array}{cc}
-3 & 1 \\
0 & 0
\end{array}\right) \otimes \mathbb{1}+\left(\begin{array}{cc}
0 & 0 \\
1 & -1
\end{array}\right) \otimes \boldsymbol{\sigma}^{1}
$$

The Hamiltonian acting on the $R=3, j=1 / 2$ wave function will be a generalization of the above operator to one acting on 40 functions. We will not give the expression here, but we analyze the spectrum of the $R=3, j=1 / 2$ sector numerically in the main text.

\section{APPENDIX B: METRIC ON THE MODULI SPACE}

To determine the one-loop effective action for the $\nu=0$ theory, we follow [28,58,59] and pass to the Lagrangian formulation of our gauge-quantum mechanics, including gauge-fixing terms and ghosts. We will use the background field method [60,61] - that is, we will expand the fields $X_{A}^{i}=B_{A}^{i}+\tilde{X}_{A}^{i}$ where $B_{A}^{i}$ is a fixed background field configuration and $\tilde{X}_{A}^{i}$ are the fluctuating degrees of freedom. We choose $B_{A}^{i}=\delta_{A 3} \overrightarrow{\mathbf{x}}$ such that it parametrizes motion along the moduli space.

The gauge-fixed Lagrangian is

$$
\mathcal{L}=\mathcal{L}_{\text {bos. }}+\mathcal{L}_{\text {ferm. }}+\mathcal{L}_{\text {g.f. }}+\mathcal{L}_{\text {ghost }}
$$

with

$$
\begin{aligned}
\mathcal{L}_{\text {bos. }}= & \frac{1}{2}\left(\mathcal{D}_{t} X_{A}^{i}\right)^{2}-\frac{g^{2}}{4}\left(f_{A B C} X_{B}^{i} X_{C}^{j}\right)^{2}, \\
\mathcal{L}_{\text {ferm. }}= & i\left(\bar{\lambda}_{A} \mathcal{D}_{t} \lambda_{A}-g f_{A B C} \bar{\lambda}_{A} X_{B}^{k} \sigma^{k} \lambda_{C}\right), \\
& \mathcal{L}_{\text {g.f. }}=-\frac{1}{2 \xi}\left(\mathcal{D}_{t}^{\mathrm{bg}} A_{A}\right)^{2}, \\
\mathcal{L}_{\text {ghost }}= & \bar{c}_{A}\left(-\delta_{A B} \partial_{t}^{2}-g f_{A C B} \partial_{t}\left(A_{C} \cdot\right)\right. \\
& \left.+g^{2} f_{A C D} f_{D E B} B_{C}^{i} X_{E}^{i}\right) c_{B},
\end{aligned}
$$

and

$$
\begin{aligned}
\mathcal{D}_{t} X_{A}^{i} & \equiv \dot{X}_{A}^{i}+g f_{A B C} A_{B} X_{C}^{i}, \quad \mathcal{D}_{t} \lambda_{A \alpha} \equiv \dot{\lambda}_{A \alpha}+g f_{A B C} A_{B} \lambda_{C \alpha}, \\
\mathcal{D}_{t}^{\mathrm{bg}} A_{A} & \equiv-\dot{A}_{A}+g f_{A B C} B_{B}^{i} X_{C}^{i} .
\end{aligned}
$$

We further set $\xi=1$, corresponding to Feynman gauge. We can obtain the correction to the metric on moduli space by choosing a background field $\overrightarrow{\mathbf{x}}$ as follows [28]:

$$
\overrightarrow{\mathbf{x}}=(b, v t, 0)
$$

where $b$ is to be thought of as an impact parameter for a particle moving at speed $v$. We now Wick rotate $t \rightarrow-i \tau$, $v \rightarrow i \gamma$, and $A_{A} \rightarrow i A_{A}$ and expand the action to quadratic order in fluctuating fields about the background field $B_{A}^{i}=\delta_{A 3} \overrightarrow{\mathbf{x}}$. The idea is to integrate out all fields that obtain a mass, through interaction with the background field, at one loop.

Following this procedure, it is easy to show that all fields with color index $A=3$ remain massless, while the rest obtain time dependent masses. After diagonalizing the mass matrix for the bosonic fields, we find the contribution to the Euclidean effective action coming, respectively, from the bosonic, fermionic, and ghost determinants:

$$
\begin{aligned}
& \delta S_{E}^{\text {bos. }}=-2 \operatorname{Tr} \log \left(-\partial_{\tau}^{2}+g^{2}\left(b^{2}+\gamma^{2} \tau^{2}\right)\right) \\
&-\operatorname{Tr} \log \left(-\partial_{\tau}^{2}+g^{2}\left(b^{2}+\gamma^{2} \tau^{2}\right)-2 g \gamma\right) \\
&-\operatorname{Tr} \log \left(-\partial_{\tau}^{2}+g^{2}\left(b^{2}+\gamma^{2} \tau^{2}\right)+2 g \gamma\right), \\
& \delta S_{E}^{\text {ferm. }}= \operatorname{Tr} \log \left(\begin{array}{cc}
\partial_{\tau} & -g(\gamma \tau+i b) \\
-g(\gamma \tau-i b) & \partial_{\tau}
\end{array}\right) \\
&+\operatorname{Tr} \log \left(\begin{array}{cc}
\partial_{\tau} & g(\gamma \tau-i b) \\
g(\gamma \tau+i b) & \partial_{\tau}
\end{array}\right), \\
& \delta S_{E}^{\text {ghost }}=2 \operatorname{Tr} \log \left(-\partial_{\tau}^{2}+g^{2}\left(b^{2}+\gamma^{2} \tau^{2}\right)\right) .
\end{aligned}
$$

Note that the ghost determinant cancels against the contribution coming from four of the eight massive bosons. Up to a diverging constant, which will cancel between the bosonic and fermionic terms, we can replace $\log (\lambda)=$ $-\int_{0}^{\infty} \frac{d s}{s} e^{-s \lambda}$ and, summing over the spectra of the above differential operators, we find 


$$
\begin{aligned}
\delta S_{E} & =\int_{0}^{\infty} \frac{d s}{s} e^{-b^{2} g^{2} s}(\cosh (2 g \gamma s) \operatorname{csch}(g \gamma s)-\operatorname{coth}(g \gamma s)) \\
& =\int_{0}^{\infty} \frac{d s}{s} e^{-b^{2} g^{2} s} \operatorname{sech}\left(\frac{g \gamma s}{2}\right) \sinh \left(\frac{3 g \gamma s}{2}\right)
\end{aligned}
$$

Let us now wick rotate back to Lorentzian time and use

$\frac{e^{-b^{2} g^{2} s}}{s}=\int \frac{d t}{\sqrt{\pi s}} g v e^{-s g^{2} r^{2}}, \quad r^{2}=b^{2}+v^{2} t^{2}$,

to write down the Lorentzian action to $O\left(v^{2}\right)$ : $i S_{L}=i \int d t\left[\frac{v^{2}}{2}-g v \int \frac{d s}{\sqrt{\pi s}} e^{-s g^{2} r^{2}} \sec \left(\frac{g v s}{2}\right) \sin \left(\frac{3 g v s}{2}\right)\right]$

$$
=i \int d t \frac{1}{2}\left(1-\frac{3}{2 g r^{3}}\right) v^{2}+O\left(v^{4}\right)
$$

which is the same correction as found in [22,23]. It also resembles the correction to the moduli space metric in the D0-D4 system [28], albeit with a different coefficient and sign.
[1] M. Claudson and M. B. Halpern, Supersymmetric ground state wave functions, Nucl. Phys. B250, 689 (1985).

[2] F. Denef, Quantum quivers and Hall/hole halos, J. High Energy Phys. 10 (2002) 023.

[3] C. T. Asplund, F. Denef, and E. Dzienkowski, Massive quiver matrix models for massive charged particles in AdS, J. High Energy Phys. 01 (2016) 055.

[4] T. Banks, W. Fischler, S. H. Shenker, and L. Susskind, M theory as a matrix model: A conjecture, Phys. Rev. D 55, 5112 (1997).

[5] D. E. Berenstein, J. M. Maldacena, and H.S. Nastase, Strings in flat space and pp waves from $N=4$ superYang-Mills, J. High Energy Phys. 04 (2002) 013.

[6] S. Sethi and M. Stern, D-brane bound states redux, Commun. Math. Phys. 194, 675 (1998).

[7] G. W. Moore, N. Nekrasov, and S. Shatashvili, D particle bound states and generalized instantons, Commun. Math. Phys. 209, 77 (2000).

[8] P. Yi, Witten index and threshold bound states of D-branes, Nucl. Phys. B505, 307 (1997).

[9] S.-J. Lee and P. Yi, Witten index for noncompact dynamics, J. High Energy Phys. 06 (2016) 089.

[10] B. de Wit, Supersymmetric quantum mechanics, supermembranes and Dirichlet particles, Nucl. Phys. B, Proc. Suppl. 56, 76 (1997).

[11] J. Polchinski, M theory and the light cone, Prog. Theor. Phys. Suppl. 134, 158 (1999).

[12] T. Anous, SUSY in Silico: Numerical D-brane bound state spectroscopy, Phys. Rev. D 94, 106014 (2016).

[13] J. Wosiek, Spectra of supersymmetric Yang-Mills quantum mechanics, Nucl. Phys. B644, 85 (2002).

[14] M. Campostrini and J. Wosiek, High precision study of the structure of $D=4$ supersymmetric Yang-Mills quantum mechanics, Nucl. Phys. B703, 454 (2004).

[15] D. Anninos, T. Anous, F. Denef, and L. Peeters, Holographic vitrification, J. High Energy Phys. 04 (2015) 027.

[16] O. Aharony, O. Bergman, D. L. Jafferis, and J. Maldacena, $\mathcal{N}=6$ superconformal Chern-Simons-matter theories,
M2-branes and their gravity duals, J. High Energy Phys. 10 (2008) 091.

[17] A. V. Smilga, Witten index calculation in supersymmetric gauge theory, Yad. Fiz. 42, 728 (1985) [Nucl. Phys. B266, 45 (1986)].

[18] J. Goldstone and R. Jackiw, Unconstrained temporal gauge for Yang-Mills theory, Phys. Lett. 74B, 81 (1978).

[19] J. Harvey, R. Iengo, K. S. Narain, S. Randjbar-Daemi, and H. Verlinde, String theory in two-dimensions, in Spring School on String Theory and Quantum Gravity (to be followed by Workshop) Trieste, Italy, 1991 (World Scientific, Singapore, 1991), pp. 30-101, https://doi.org/ $10.1142 / 1494$.

[20] M. B. Halpern and C. Schwartz, Asymptotic search for ground states of SU(2) matrix theory, Int. J. Mod. Phys. A 13, 4367 (1998).

[21] M. J. Duff, C. N. Pope, and E. Sezgin, Supermembranes and Physics in 2+1 Dimensions (World Scientific, Singapore, 1990), https://doi.org/10.1142/1028.

[22] A. V. Smilga, Born-Oppenheimer corrections to the effective zero mode Hamiltonian in SYM theory, J. High Energy Phys. 04 (2002) 054.

[23] E. T. Akhmedov and A. V. Smilga, On the relation between effective supersymmetric actions in different dimensions, Yad. Fiz. 66, 2290 (2003) [Phys. At. Nucl. 66, 2238 (2003)].

[24] J. Frohlich, G. M. Graf, D. Hasler, J. Hoppe, and S.-T. Yau, Asymptotic form of zero energy wave functions in supersymmetric matrix models, Nucl. Phys. B567, 231 (2000).

[25] G. M. Graf and J. Hoppe, Asymptotic ground state for 10-dimensional reduced supersymmetric SU(2) Yang-Mills theory, arXiv:hep-th/9805080.

[26] E. A. Ivanov and A. V. Smilga, Supersymmetric gauge quantum mechanics: Superfield description, Phys. Lett. B 257, 79 (1991).

[27] D.-E. Diaconescu and R. Entin, A Nonrenormalization theorem for the $d=1, N=8$ vector multiplet, Phys. Rev. D 56, 8045 (1997). 
[28] M. R. Douglas, D. N. Kabat, P. Pouliot, and S. H. Shenker, D-branes and short distances in string theory, Nucl. Phys. B485, 85 (1997).

[29] D. Anninos, T. Anous, P. de Lange, and G. Konstantinidis, Conformal quivers and melting molecules, J. High Energy Phys. 03 (2015) 066.

[30] Y.-H. Lin and X. Yin, On the ground state wave function of matrix theory, J. High Energy Phys. 11 (2015) 027.

[31] W. Taylor, M(atrix) theory: Matrix quantum mechanics as a fundamental theory, Rev. Mod. Phys. 73, 419 (2001).

[32] L. Susskind, Holography in the flat space limit, AIP Conf. Proc. 493, 98 (1999).

[33] V. Balasubramanian, R. Gopakumar, and F. Larsen, Gauge theory, geometry and the large $\mathrm{N}$ limit, Nucl. Phys. B526, 415 (1998).

[34] D. Anninos, T. Anous, and F. Denef, Disordered quivers and cold horizons, J. High Energy Phys. 12 (2016) 071.

[35] A. Kitaev, A simple model of quantum holography, KITP strings seminar and Entanglement program, 2015, http:// online.kitp.ucsb.edu/online/entangled15/.

[36] J. Polchinski and V. Rosenhaus, The spectrum in the Sachdev-Ye-Kitaev model, J. High Energy Phys. 04 (2016) 001.

[37] J. Maldacena and D. Stanford, Remarks on the Sachdev-YeKitaev model, Phys. Rev. D 94, 106002 (2016).

[38] K. Jensen, Chaos in $\mathrm{AdS}_{2}$ Holography, Phys. Rev. Lett. 117, 111601 (2016).

[39] S. Sachdev, Bekenstein-Hawking Entropy and Strange Metals, Phys. Rev. X 5, 041025 (2015).

[40] A. Jevicki, K. Suzuki, and J. Yoon, Bi-local holography in the SYK model, J. High Energy Phys. 07 (2016) 007.

[41] J. S. Cotler, G. Gur-Ari, M. Hanada, J. Polchinski, P. Saad, S. H. Shenker, D. Stanford, A. Streicher, and M. Tezuka, Black holes and random matrices, J. High Energy Phys. 05 (2017) 118.

[42] E. Witten, An SYK-Like model without disorder, arXiv: 1610.09758 .

[43] R. Gurau, The complete $1 / N$ expansion of a SYK-like tensor model, Nucl. Phys. B916, 386 (2017).

[44] I. R. Klebanov and G. Tarnopolsky, Uncolored random tensors, melon diagrams, and the SYK models, Phys. Rev. D 95, 046004 (2017).
[45] C. Krishnan, S. Sanyal, and P. N. Bala Subramanian, Quantum Chaos and holographic tensor models, J. High Energy Phys. 03 (2017) 056.

[46] J. Maldacena, S. H. Shenker, and D. Stanford, A bound on chaos, J. High Energy Phys. 08 (2016) 106.

[47] D. Anninos and G. A. Silva, Solvable quantum Grassmann matrices, J. Stat. Mech. (2017) 043102.

[48] F. Ferrari, The large D limit of planar diagrams, arXiv: 1701.01171.

[49] G. Gur-Ari, M. Hanada, and S. H. Shenker, Chaos in classical D0-brane mechanics, J. High Energy Phys. 02 (2016) 091.

[50] B. Balthazar, V. A. Rodriguez, and X. Yin, Hamiltonian truncation study of supersymmetric quantum mechanics: S-Matrix and metastable states, arXiv:1610.07275.

[51] R. C. Ferrell and D. M. Eardley, Slow Motion Scattering and Coalescence of Maximally Charged Black Holes, Phys. Rev. Lett. 59, 1617 (1987).

[52] J. Michelson and A. Strominger, The geometry of (super) conformal quantum mechanics, Commun. Math. Phys. 213, 1 (2000).

[53] J. Michelson and A. Strominger, Superconformal multiblack hole quantum mechanics, J. High Energy Phys. 09 (1999) 005.

[54] R. Britto-Pacumio, J. Michelson, A. Strominger, and A. Volovich, Lectures on superconformal quantum mechanics and multi-black hole moduli spaces, NATO Sci. Ser. C 556, 255 (2000).

[55] G. M. Asatryan and G. K. Savvidy, Configuration manifold of Yang-Mills classical mechanics, Phys. Lett. 99A, 290 (1983).

[56] G. K. Savvidy, Yang-Mills classical mechanics as a Kolmogorov K system, Phys. Lett. 130B, 303 (1983).

[57] G. K. Savvidy, Yang-Mills quantum mechanics, Phys. Lett. 159B, 325 (1985).

[58] K. Becker and M. Becker, A two loop test of M(atrix) theory, Nucl. Phys. B506, 48 (1997).

[59] W. Taylor and M. Van Raamsdonk, Supergravity currents and linearized interactions for matrix theory configurations with fermionic backgrounds, J. High Energy Phys. 04 (1999) 013.

[60] L. F. Abbott, Introduction to the background field method, Acta Phys. Pol. B 13, 33 (1982).

[61] L. F. Abbott, The background field method beyond one loop, Nucl. Phys. B185, 189 (1981). 